INL/EXT-15-36779 Rev. 0

September 2015

\title{
Summary of BISON Development and Validation Activities - NEAMS FY15 Report
}

\author{
R. L. Williamson \\ K. A. Gamble \\ S. R. Novascone \\ W. Liu \\ B. W. Spencer \\ G. Pastore \\ J. D. Hales \\ R. J. Gardner \\ D. M. Perez
}

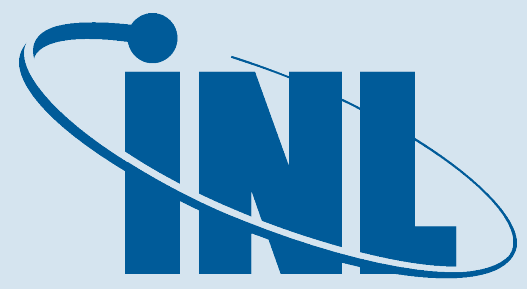

Idaho National Laboratory 


\section{NOTICE}

This information was prepared as an account of work sponsored by an agency of the U.S. Government. Neither the U.S. Government nor any agency thereof, nor any of their employees, makes any warranty, express or implied, or assumes any legal liability or responsibility for any third party's use, or the results of such use, of any information, apparatus, product, or process disclosed herein, or represents that its use by such third party would not infringe privately owned rights. The views expressed herein are not necessarily those of the U.S. Nuclear Regulatory Commission. 
INL/EXT-15-36779 Rev. 0

Summary of BISON Development and Validation Activities NEAMS FY15 Report

\author{
R. L. Williamson \\ K. A. Gamble \\ S. R. Novascone \\ W. Liu* \\ B. W. Spencer \\ G. Pastore \\ J. D. Hales \\ R. J. Gardner \\ D. M. Perez
}

${ }^{*}$ ANATECH, Corp.

September 2015

\title{
Idaho National Laboratory
}

Fuel Modeling and Simulation Department

Idaho Falls, Idaho 83415

\author{
Prepared for the \\ U.S. Department of Energy \\ Office of Nuclear Energy \\ Under U.S. Department of Energy-Idaho Operations Office \\ Contract DE-AC07-99ID13727
}




\section{Contents}

\begin{tabular}{lll}
\hline & Introduction & 6
\end{tabular}

\begin{tabular}{|lll}
2 & Milestone Summary & $\mathbf{7}$
\end{tabular}

$2.1 \quad$ Milestone Level and Completion Schedule . . . . . . . . . . . . . . . . . . 7

2.2 Release BISON Update for LWR Fuel Performance in Quasi-Steady, Off-Normal

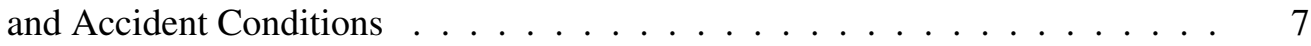

2.3 Issue Update to BISON Validation Report . . . . . . . . . . . . . . . . 8

2.4 Demonstrate Coupling of RELAP7 and BISON $\ldots \ldots \ldots \ldots \ldots$

2.5 Convert Selected ENIGMA Validation Input Files to BISON Input Files and Run

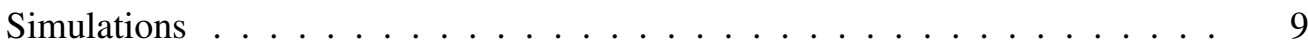

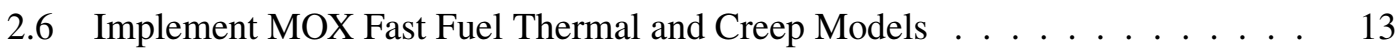

2.6 .1 Thermal Conductivity . . . . . . . . . . . . . . . 13

$2.6 .2 \quad$ Steady State Thermal and Irradiation Creep . . . . . . . . . . . . . . . 13

2.7 Improvements to the Coolant Channel Model $\ldots \ldots \ldots$. . . . . . . . . . . . . . 14

$2.7 .1 \quad$ Code optimization $\ldots \ldots \ldots \ldots \ldots$. . . . . . . . . . . . . . . 14

$2.7 .2 \quad$ Improvements to the LOCA reflood model $\ldots \ldots \ldots \ldots$. . . . . . . . 14

2.7 .3 Additions for fast reactor behavior . . . . . . . . . . . . . . . . . . 17

3 Additional Major Accomplishments 18

$3.1 \quad$ BISON Validation Summary Article $\ldots \ldots \ldots$. . . . . . . . . . . . . 18

3.1 .1 Verification . . . . . . . . . . . . . . . . . 18

$3.1 .2 \quad$ Experimental data used for validation . . . . . . . . . . . . . 18

$3.1 .3 \quad$ Thermal Behavior $\ldots \ldots \ldots \ldots 19 . \ldots \ldots$

3.1 .4 Fission Gas Release $\ldots \ldots \ldots \ldots \ldots$

3.1 .5 Rod Diameter . . . . . . . . . . . . . . . . . . . . . . 21

3.1 .6 Summary . . . . . . . . . . . . . . . . . . . . . . . . . . . 23

3.2 Multiphysics Coupling Article . . . . . . . . . . . . . . . . . . . . 24

3.2 .1 Thermomechanical contact example . . . . . . . . . . . . 25

3.2 .2 LWR fuel performance simulation example . . . . . . . . . . . . . . 27

3.2 .3 Conclusions . . . . . . . . . . . . . . . . . . . 30

3.3 Reactivity Insertion Accident Analysis $\ldots \ldots \ldots$. . . . . . . . . . . . . . . . . . . 30

3.4 Uncertainty Analysis in Fuel Modeling . . . . . . . . . . . . . . . . . . 31

\begin{tabular}{|lll}
\hline 4 & Future Work & 36
\end{tabular}

\begin{tabular}{lll}
\hline 5 & Acknowledgements & 37
\end{tabular} 


\section{Introduction}

The objective of the NEAMS ToolKit is to develop a "pellet-to-plant" simulation capability useful for predicting performance and safety for a broad range of nuclear reactor power systems. The NEAMS ToolKit has been organized into a Fuels Product Line (FPL) and a Reactor Product Line (RPL) and is modular in design. Within the FPL, a multiscale approach has been adopted in which simulations of fuel performance at the engineering scale are informed by material property and irradiation performance models developed from mesoscale simulations of microstructural evolution. The focus in this report is on development and validation of the engineering-scale fuel performance analysis tool within the FPL, which is BISON [1].

This summary report contains an overview of work performed under the work package entitled "FY2015 NEAMS INL-Engineering Scale Fuel Performance (BISON)" A first chapter identifies the specific FY-15 milestones, providing a basic description of the associated work and references to related detailed documentation. Where applicable, a representative technical result is provided. A second chapter summarizes major additional accomplishments including 1) a BISON validation paper, 2) a multiphysics coupling paper, 3) application of BISON to Reactivity Insertion Accident analysis and 4) use of BISON for uncertainty analysis in fuel modeling. A final chapter outlines FY-16 future work. 


\section{Milestone Summary}

\subsection{Milestone Level and Completion Schedule}

FY-2015 Milestones and the completion dates are listed in Table 2.1. Following sections contain a short description of each milestone and references to related detailed documentation. Where applicable, a representative technical result from the work is included.

Table 2.1: FY-2015 Milestones for NEAMS INL-Engineering Scale Fuel Performance Effort

\begin{tabular}{lcc}
\hline Milestone & Completion Date & MS Level \\
\hline $\begin{array}{l}\text { Release BISON update for LWR fuel performance in } \\
\text { quasi-steady, off-normal and accident conditions. }\end{array}$ & $9 / 30$ & M2 \\
\hline Issue update to BISON validation and assessment report & $9 / 30$ & M2 \\
\hline Demonstrate coupling RELAP7 to BISON & $2 / 28$ & M3 \\
\hline $\begin{array}{l}\text { Convert selected ENIGMA validation input files } \\
\text { to BISON input files and run simulations }\end{array}$ & $4 / 30$ & M3 \\
\hline Implement MOX fast fuel thermal and creep models & $6 / 30$ & M3 \\
\hline Improvements to the coolant channel model & $9 / 30$ & M3 \\
\hline
\end{tabular}

\subsection{Release BISON Update for LWR Fuel Performance in Quasi-Steady, Off-Normal and Accident Conditions}

The major accomplishment for this year was the release of an updated version of BISON (Version 1.2) with corresponding documentation including an updated user [2] and theory manual [3]. The BISON training materials were also significantly improved to include more basic thermomechanics example problems and more detailed information on fuels-specific models. The major new or improved capabilities in Version 1.2 include:

- Coolant channel model improvements including:

LOCA reflood capability

Applicability to sodium coolant and hexagonal channel geometries

Performance optimization

- Nonlinear mechanics models were updated to handle large rotations that occur in clad during LOCAs (ballooning) 
- High temperature burst model for Zircaloy under accident conditions

- Thermal and creep models for MOX (30\% Pu) fuel

- Improved mechanical contact performance and robustness

- Improved frictional contact

- Capability to map 2D-RZ results for a full-length fuel rod to a 3D submodel

- Material models for U-Pu-Zr metal fuel and HT-9 steel cladding (this development was funded principally by the Advanced Fuel Campaign)

Near the end of FY-14, BISON was migrated to a new code repository (GitLab) and configuration management tool (git). This new approach provides the development team with a mechanism to review, approve or disapprove, and document all changes to the BISON source code. This tool has proven extremely useful in enforcing BISON software quality standards. Software must be well-written and accompanied by appropriate regression testing and documentation before it is included in the code repository.

\subsection{Issue Update to BISON Validation Report}

The BISON Light Water Reactor (LWR) validation base was significantly enlarged by adding separate effects experiments and additional cases from the FUMEX-II [4] and FUMEX-III [5] international coordinated research projects. The validation base was further enlarged by comparison to 13 commercial fuel rods. These new cases and all cases considered to date are documented in the updated BISON Validation report [6].

\subsection{Demonstrate Coupling of RELAP7 and BISON}

As mentioned above, fuel performance modeling in BISON is currently being expanded to include loss of coolant accidents (LOCA). The effects of LOCA on the fuel rod include rapid oxidation of the cladding, creep and rupture of the cladding, and overheating of the fuel pellets. These occur because of dramatic reduction of heat transfer into the coolant due to boil off of the coolant. The BISON coolant channel model currently contains a simplistic LOCA analysis capability based on a simple 1D enthalpy balance and includes boiling curves and convective heat transfer correlations for various flow regimes. It is expected that much improved simulations will be realized by coupling BISON to the advanced multiphase flow code RELAP-7, which is under active development at INL. This milestone was designed to provide an early demonstration of this coupled capability.

Both BISON and RELAP-7 are based on the MOOSE finite element computational framework. MOOSE includes automatic transfers of variables and coupled time stepping that enable a MOOSE-based application to easily control other MOOSE-based applications while transferring field data through memory (i.e. without resorting to file-based transfers). Fields and scalar 
variables are transferred via MOOSE's Transfer feature, which allows various types of interpolations, projections, or other evaluations of data that are to be moved between the coupled applications. Control of tight versus loose coupling and sequencing of the applications is by MOOSE's MultiApp feature. The coupling scheme and transfer of variables is easily controlled from the input file so that various schemes can be quickly investigated.

This milestone was completed by coupling BISON (in 2D or 3D) to a 1D pipe flow with RELAP-7. The coupling is contained within the MAMMOTH application. The coupled variables currently include cladding surface temperature, heat transfer coefficients along the cladding, and bulk coolant temperature. To enable LOCA simulations, 2-phase fluid flow has been assumed, so there are extra variables for vapor and liquid phase fractions as well as separate vapor and liquid heat transfer coefficients.

To demonstrate coupling, an AP-1000 type fuel rod was coupled to a 1D pipe model for the coolant. The AP-1000 rod has dual upper and lower plenums with a fuel stack $4.3 \mathrm{~m}$ tall. The linear heat generation rate was assumed to be $17.5 \mathrm{~kW} / \mathrm{m}$ for a 4 -year irradiation. The BISON model includes thermo-mechanical contact. Two-phase flow is computed by RELAP-7 for a channel with pin pitch of $1.26 \mathrm{~mm}$ and a rod diameter of $9.5 \mathrm{~mm}$. Inlet flow is $3269 \mathrm{~kg} / \mathrm{m} 2-\mathrm{s}$ at $553 \mathrm{~K}$. The computed axial profiles of temperature and vapor fraction of the fluid are shown in Figure 2.1. The coolant begins to boil slightly (nucleate boiling) near the middle of the rod. Currently, the equation of state in RELAP-7 does not contain valid parameters for this regime, so the pressure is approximately $20 \mathrm{MPa}$ (rather than the $15.5 \mathrm{MPa}$ design pressure in AP-1000). The thermodynamics in RELAP-7 are being actively developed, and this situation will improve soon.

\subsection{Convert Selected ENIGMA Validation Input Files to BISON Input Files and Run Simulations}

BISON is currently being validated to both separate effects experiments and a wide variety of LWR integral fuel rod experiments. Validation to integral fuel rod experiments is often difficult and time consuming due to the complexity of both the experiments and associated models. Leveraging existing high quality validation efforts from other fuel performance codes offers the promise of streamlining BISON validation efforts.

ENIGMA is an internationally known fuel performance code that was developed at the National Nuclear Laboratory (NNL) in the UK and has been in use for decades. ENIGMA has been validated against approximately 540 fuel performance experiments. As part of an INL/NNL collaborative agreement, NNL has offered access to experimental data and input files for roughly 220 of these cases. Efforts have begun to develop a process for conversion of these cases to expedite BISON validation.

To achieve this milestone, the BISON team successfully converted two LWR cases: 1) Halden IFA-515 Rod A1 and 2) OSIRIS Rod J12. Note that, by design, initial conversion efforts are on cases previously analyzed by the BISON validation team. This provided an in-depth examination of the input data conversion process on well-known experiments. Additionally it provides an opportunity for code-to-code comparison and comparisons to experimental data. A few simple examples of comparing the original BISON simulation to the ENIGMA-based BISON simula- 


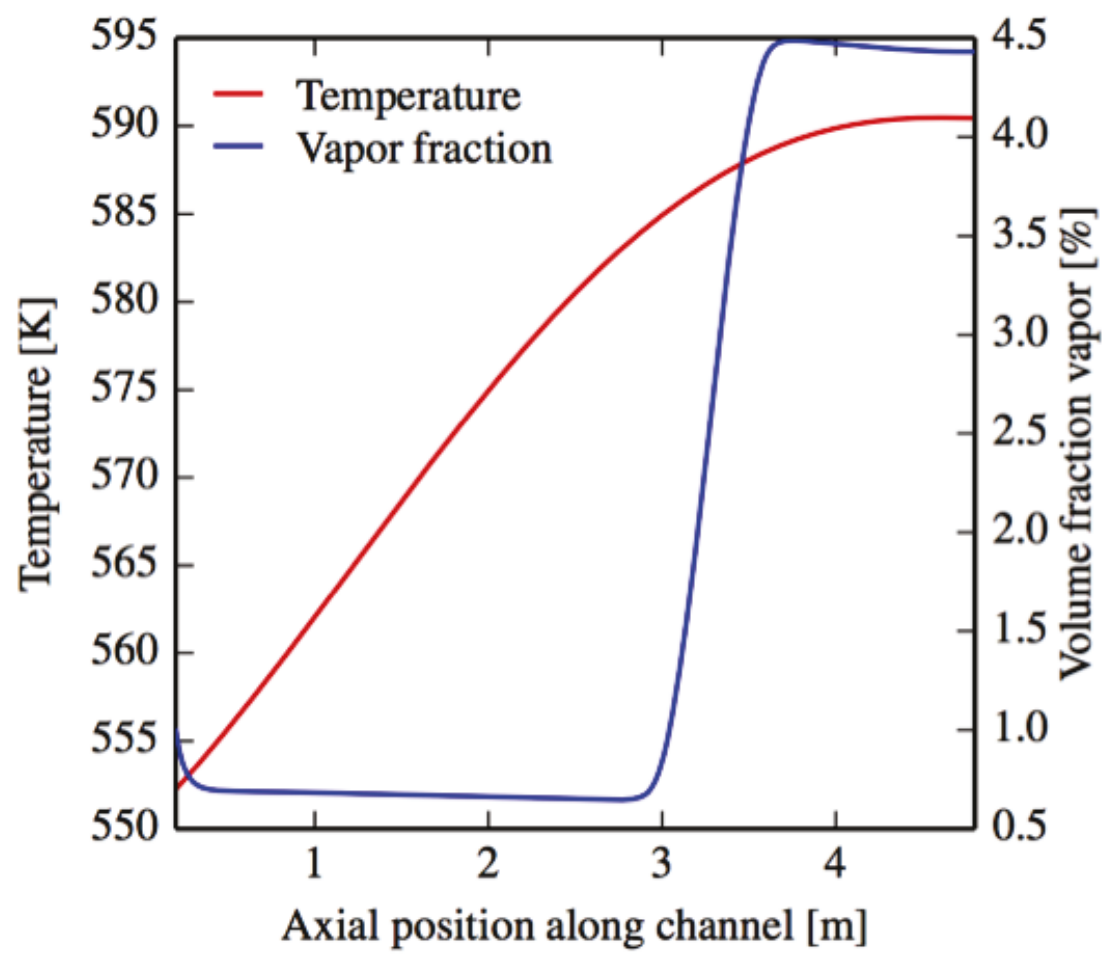

Figure 2.1: The axial profiles of temperature and vapor fraction from the coupled BISON/RELAP-7 simulation of an AP1000 fuel rod. 
tion are shown below.

IFA-515 Rod A1 is used to validate beginning of life and through life fuel behavior. Figure 2.2 shows the fuel linear heat rate as measured in the experiment, as used in the original BISON validation calculations, and as input to BISON using input data from the corresponding ENIGMA validation case. Comparisons are good, demonstrating success in interpreting and converting an ENIGMA power history to a BISON power history.

OSIRIS Rod J12 is used to validate the pellet-clad mechanical interface (PCMI) at the end of life, by ramp testing a fuel rod segment that was initially irradiated in a commercial reactor. Figure 2.3 shows fuel centerline temperature histories for the original BISON simulation and the ENIGMA-based BISON simulation. The pellets in the original BISON simulation were modeled as discrete where the ENIGMA-based simulation had smeared pellets. When discrete pellet geometry is used, the temperature varies from the middle to the end of the pellet, and is the reason for undulations in temperature vs. axial location for the original BISON case. Comparisons are very good, demonstrating success in conversion of the ENIGMA validation case to a BISON case.

Going forward, the goal is to develop an automated conversion process that will allow efficient completion of numerous LWR validation cases.

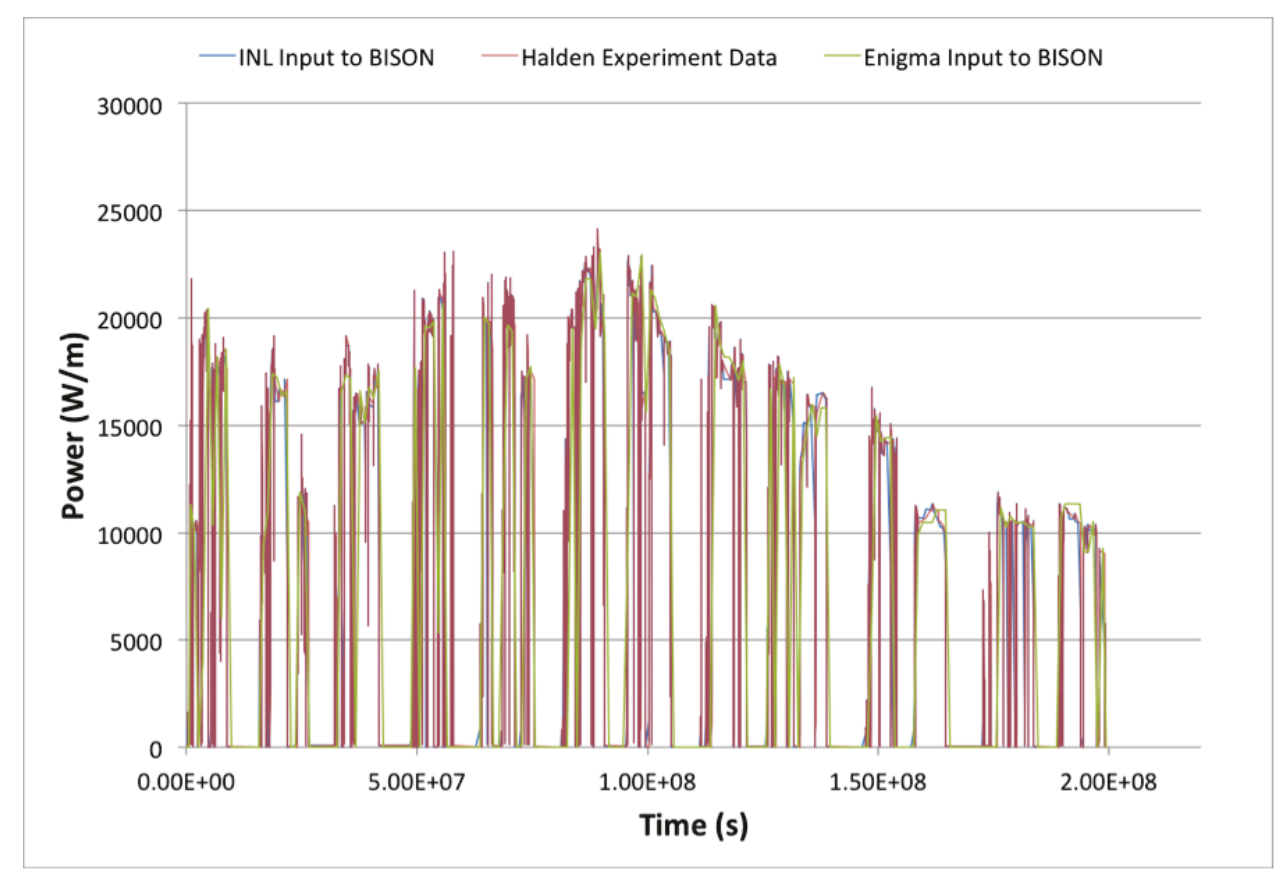

Figure 2.2: Power history comparison for the IFA-515 validation case. 


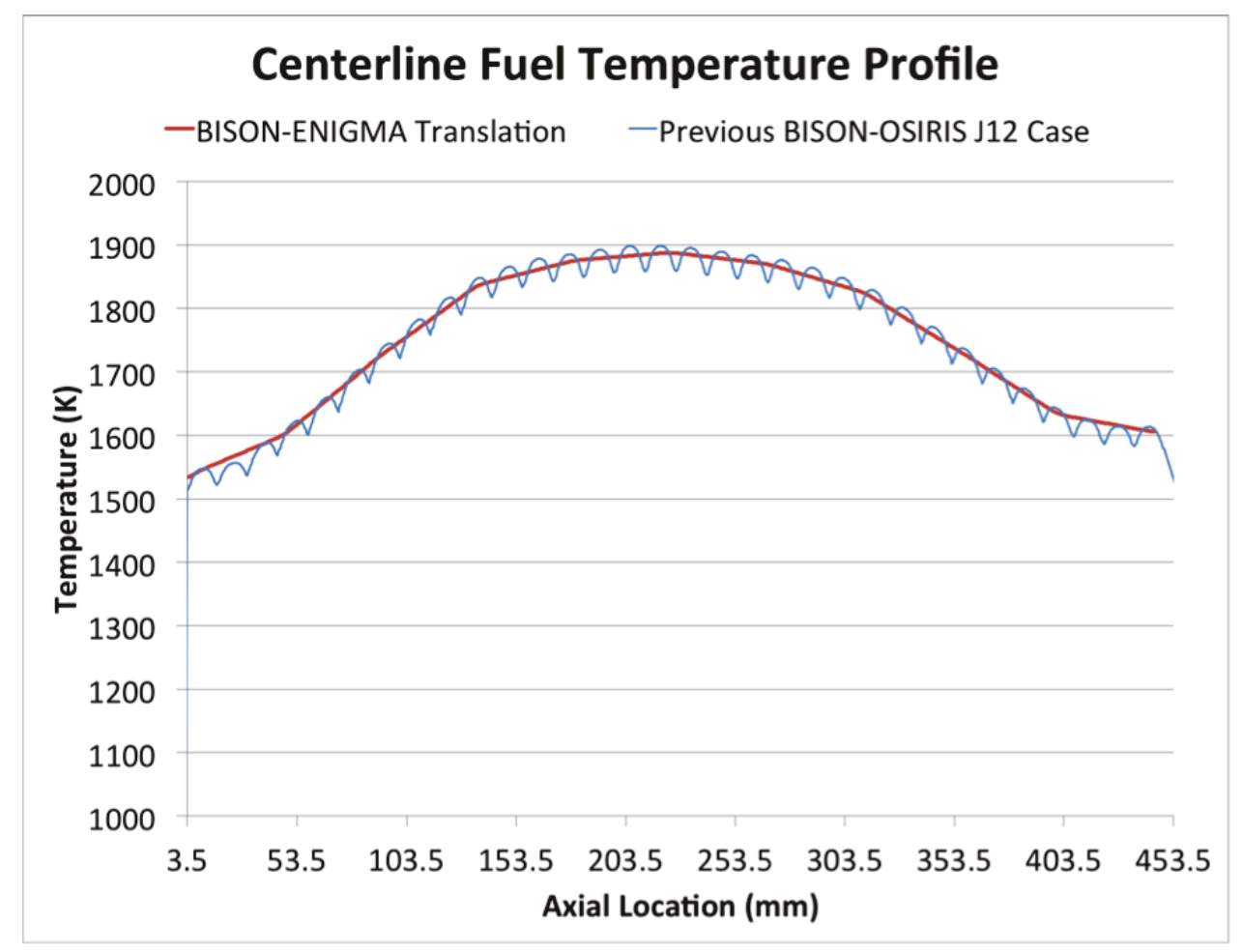

Figure 2.3: Comparison of the computed fuel centerline temperature histories from the original BISON validation calculation (discrete fuel pellets) and the BISON simulation using input data from the ENIGMA validation case (smeared fuel column). 


\subsection{Implement MOX Fast Fuel Thermal and Creep Models}

Empirical models for thermal conductivity and thermal and irradiation creep of MOX fast fuel were implemented in BISON, including regression tests and documentation in both the theory and user manuals. The specific models are described below.

\subsubsection{Thermal Conductivity}

Mixed oxide fuels for fast reactors contain higher concentrations of plutonium oxide than their LWR counterparts. The thermal conductivity model developed by Inoue et. al. [7] and used by Karahan [8] is valid for for $25 \% \mathrm{PuO}_{2}$. The thermal conductivity model for fast MOX is similar in form to the model proposed by Lucuta et. al. [9] for $\mathrm{UO}_{2}$. The model consists of an unirradiated thermal conductivity that is multiplied by corrective factors for dissolved solid fission products $\left(F_{1}\right)$, precipitated solid fission products $\left(F_{2}\right)$, radiation damage $\left(F_{3}\right)$, and porosity $\left(F_{4}\right)$ as given by:

$$
k=F_{1} F_{2} F_{3} F_{4} k_{0}
$$

where $k$ is the effective fuel thermal conductivity in $\mathrm{W} / \mathrm{m}-\mathrm{K}$ and $k_{0}$ is the fully dense fuel thermal conductivity in W/m-K. $F_{1}, F_{2}$, and $F_{3}$ are from Lucuta [9]. The equation for $F_{4}$ is the modified Loeb correlation given by:

$$
F_{4}=1-\alpha P
$$

where $P$ is the volume fraction of porosity and $\alpha$ is a coefficient. Karahan suggests a value of 2.5 for $\alpha$ for conservatism.

\subsubsection{Steady State Thermal and Irradiation Creep}

A steady-state thermal and irradiation creep model for fast MOX comes from an article by J. L. Routbort [10], described by the following equation.

$$
\begin{aligned}
\dot{\varepsilon}_{\text {thermal }+ \text { irradiation }}= & \frac{A}{G^{2}} \sigma \exp \left(-\frac{Q_{1}}{R T}\right)+B \sigma^{4.4} \exp \left(-\frac{Q_{2}}{R T}\right) \\
& +C \sigma \dot{F}
\end{aligned}
$$

where

$$
\begin{aligned}
& R=\text { Universal gas constant } 1.987(\mathrm{cal} / \mathrm{mol}-\mathrm{K}) \\
& T=\text { Temperature }(\mathrm{K}) \\
& \sigma=\text { Effective stress }(\mathrm{Pa}) \\
& \dot{F}=\text { Fission rate } \mathrm{m}^{-3} \cdot \mathrm{s}^{-1} \\
& G=\text { grain size }(\mu \mathrm{m})
\end{aligned}
$$


where the following are creep coefficients and activation energies

$$
\begin{aligned}
& A=3.23 \cdot 10^{9} \\
& B=3.24 \cdot 10^{6} \\
& C=1.78 \cdot 10^{-26} \\
& Q_{1}=92500(\mathrm{cal} / \mathrm{mol}) \\
& Q_{2}=136800(\mathrm{cal} / \mathrm{mol})
\end{aligned}
$$

\subsection{Improvements to the Coolant Channel Model}

Nuclear fuel rods are surrounded by flowing coolant which carries the thermal energy generated from the nuclear fission reactions and transfers it to a heat exchanger or turbine. To predict the thermal behavior of a fuel rod, thermal hydraulic boundary conditions of the surrounding coolant must to be determined. This can be done either by coupling to a separate flow analysis, as described in section 2.4 or via a more simplistic 1D coolant channel native to BISON. During FY-15 the existing 1D BISON coolant channel model was updated in a variety of ways to improve performance or extend applicability. This work is summarized in this section.

\subsubsection{Code optimization}

Optimization of the coolant channel models was done by adjusting the logic of calling heat transfer correlations in the coolant channel mode, and adding an option to allow users to turn on/off (model_post_chf = true or false; default=true) the post-CHF correlations. This allows the user to skip post-CHF calculations inside the code when only pre-CHF heat transfer regimes are needed. According to recent calculations, the computational time using the coolant channel model for a small problem and an AREVA benchmark case are only $11 \%$ to $13 \%$ longer, respectively, than a simple fixed convective boundary condition. Therefore, major efforts were devoted to the improvement of the functionalities of the coolant channel model with a particular emphasis on the reflooding correlation which is of interest in modeling fuel rod response during LOCA conditions.

\subsubsection{Improvements to the LOCA reflood model}

In FY-14 the FLECHT correlation, based on the equations described in [11], was implemented in BISON and documented in the BISON theory manual. FY-15 activities included further examination and testing of the FLECHT correlation, improvement to the code to address the "negative heat transfer coefficient" found in testing the correlation, and code modifications to provide a user interface to model reflooding and quenching of full length fuel rods during postulated LOCA conditions.

Negative heat transfer coefficients were found in the applicable range using the old reflood correlation in BISON. This was found in testing the correlation in one of the FLECHT reflooding tests (mid-height location in run 0791) under low flooding, low subcooling conditions. With 
comparison to the correlations in the literature, changes were made in the code to add the reflooding correlation used in [12], and the new correlation predicts more smooth changes of the heat transfer coefficient and no negative heat transfer coefficient. Results from both the new and old correlation, in comparison to measurement data from experiment 0791, are shown in Figure 2.4.

Run 0791- Reflood Heat Transfer Coefficient

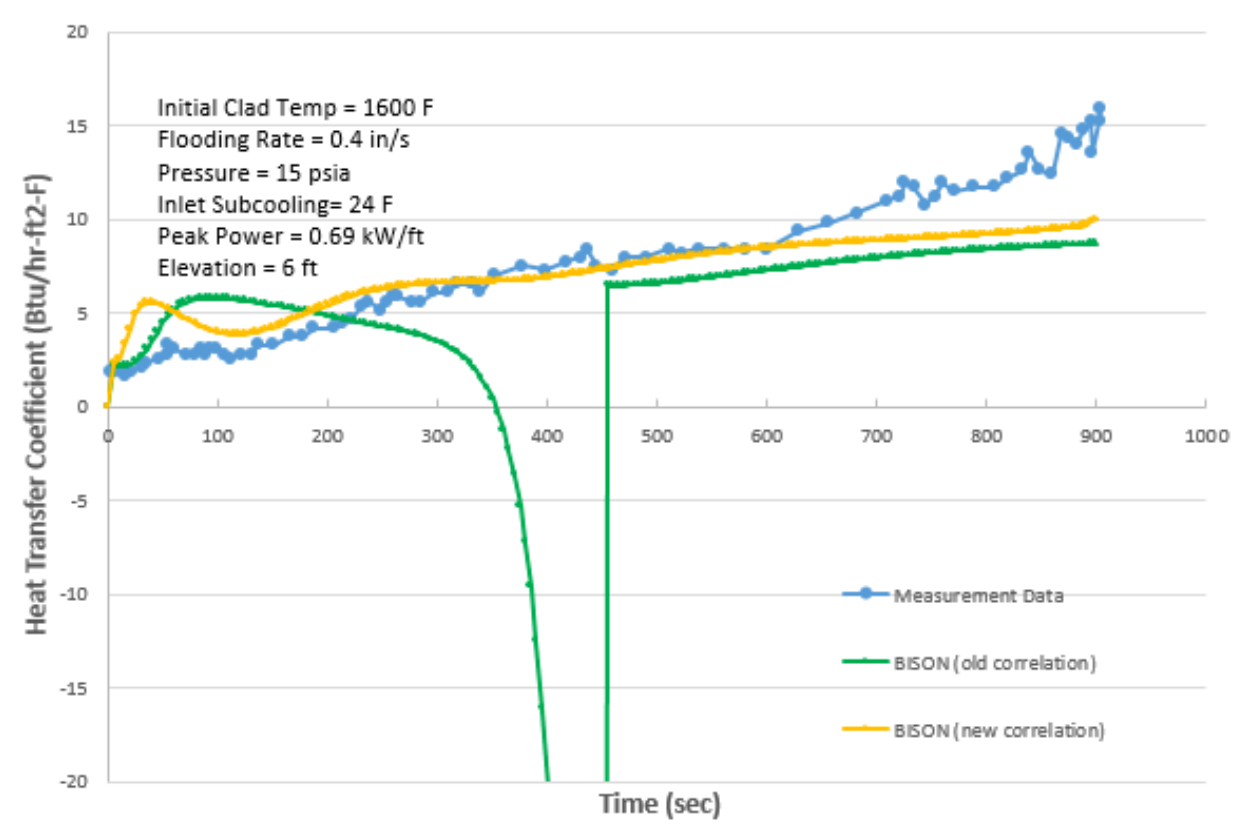

Figure 2.4: Results from both the new and old FLECHT correlations, in comparison to measured data for FLECHT experiment 0791.

Another test case, shown in Figure 2.5, further demonstrates that the new correlation tends to agree better with measurement data than the old correlation. As an input option, either the new or old correlation can be used in BISON; however, users should be cautious using the old correlation. At present time, no attempt had been made to revise or correct the old correlation in the code.

Note that the measurement data of heat transfer coefficients in Figures 2.4 and 2.5 were deduced from measured cladding temperature histories according to [12]. The large change in the coefficient at approximately $220 \mathrm{~s}$ is due to quenching, which is not currently captured in the reflooding correlation.

The FLECHT correlations only provide quench times at the fuel rod mid-height position; to model the quenching of a full length fuel rod such information is not sufficient. Simple logic based on the combination of an input rewetting temperature and the quench time predicted at the mid height position was implemented to turn on quenching. Numerical convergence has been tested and demonstrated for the dramatic change of the heat transfer coefficients. 


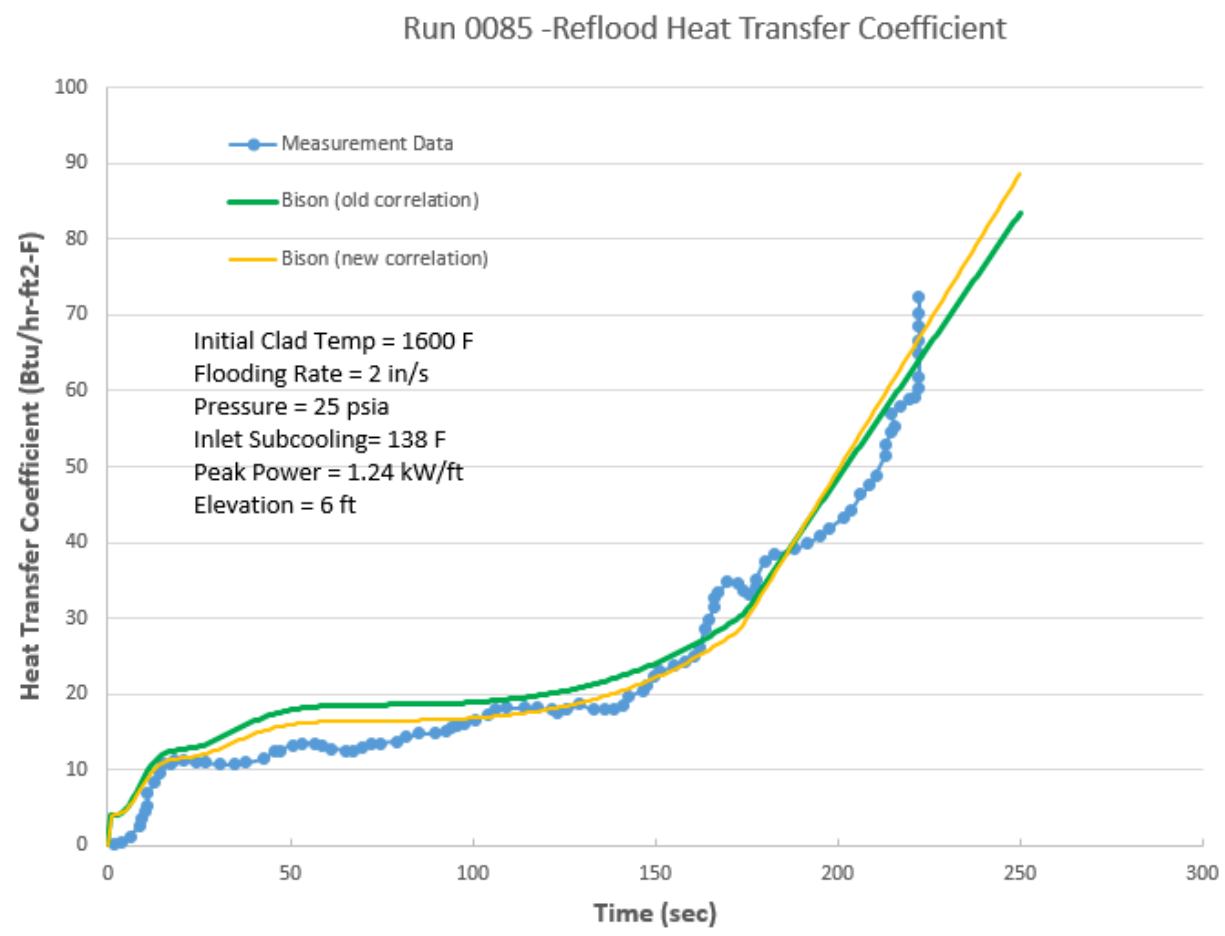

Figure 2.5: Results from both the new and old FLECHT correlations, in comparison to measured data for FLECHT experiment 0085. 
Two regression test cases (using a single element) were added to the code repository to test the FLECHT heat transfer correlations with different input options. Additionally, a benchmark case was developed and checked into the code repository. Changes to the correlation and the user interface were documented in the BISON theory and user manuals.

\subsubsection{Additions for fast reactor behavior}

The capability to use sodium coolant for fast reactors was also implemented. The model uses the same framework as for calculations of water/steam, but with an appropriate correlation for liquid sodium. The model uses the modified Schad correlation

$$
N u=4.496\left(-16.15+24.96\left(\frac{P}{D}\right)-8.55\left(\frac{P}{D}\right)^{2}\right) \frac{P e}{150}^{0.3}
$$

where

$N u \equiv h D / k$ is the Nusselt number

$P e \equiv R e P r$ is the Peclet number

$P / D$ is the pitch-to-diameter ratio

Sodium properties are taken from the ANL/RE-92/2 report [13]:

$$
\begin{aligned}
k & =124.67-0.11381 T+5.5226 \cdot 10^{-5} T^{2}-1.1842 \cdot 10^{-8} T^{3} \\
H & =-365770+1658.2 T-0.42395 T^{2}+1.4847 \cdot 10^{-4} T^{3}+2992600 / T
\end{aligned}
$$

where $k$ is thermal conductivity, $H$ is enthalpy, and units are SI.

Further, an option was implemented to simulate hexagonal coolant channel geometries. 


\section{Additional Major Accomplishments}

\subsection{BISON Validation Summary Article}

FY-15 activities included a substantial effort to develop a summary article of BISON LWR validation activities. A manuscript is nearly completed and will soon be submitted to the Journal of Nuclear Materials. The paper begins with a brief overview of BISON's computational framework, governing equations, and general material and behavioral models. Both code and solution verification are described. The validation cases considered to date are identified, and specific models and material properties used throughout the validation process are given. Results are consolidated to provide an overall view of how the code is predicting physical behavior. A brief summary of the key results from that article are provided here.

\subsubsection{Verification}

An essential prerequisite to any validation work is code and solution verification. BISON code verification is principally done using an extensive set of code regression tests, that are exercised every time a modification is proposed to the code. The structure and several examples of the BISON code verification process are given in [14].

Solution verification is also essential, to demonstrate that numerical solutions have adequate spatial and temporal resolution for the set of validation problems considered. To demonstrate this a prototypical validation problem was constructed using the set of material and behavior models employed in the validation cases, including boundary conditions and a typical power history. The problem was then run using a series of computational meshes and time increments to observe numerical convergence. Results from the spatial resolution study are shown in Figure 3.1. where relative percent error is presented for four metrics of interest: Power, Fuel Centerline Temperature (FCT), Fission Gas Release (FGR), and Rod diameter. Results are given as a function of the total number of radial elements in the mesh, where the relative error is with respect to the finest mesh considered. All LWR validation problems were run using the finest mesh shown in the figure demonstrating adequate mesh resolution for the validation study. Similar results are provided in the manuscript for temporal numerical resolution.

\subsubsection{Experimental data used for validation}

Table 3.1 summarizes the experimental fuel rods that have been used for BISON LWR validation to date. Measured quantities include fuel centerline temperature (FCT), fission gas release (FGR), and cladding outer diameter (Rod Dia) both before and following fuel-clad mechanical contact. Many of these integral rod experiments were chosen based on INL participation in the 


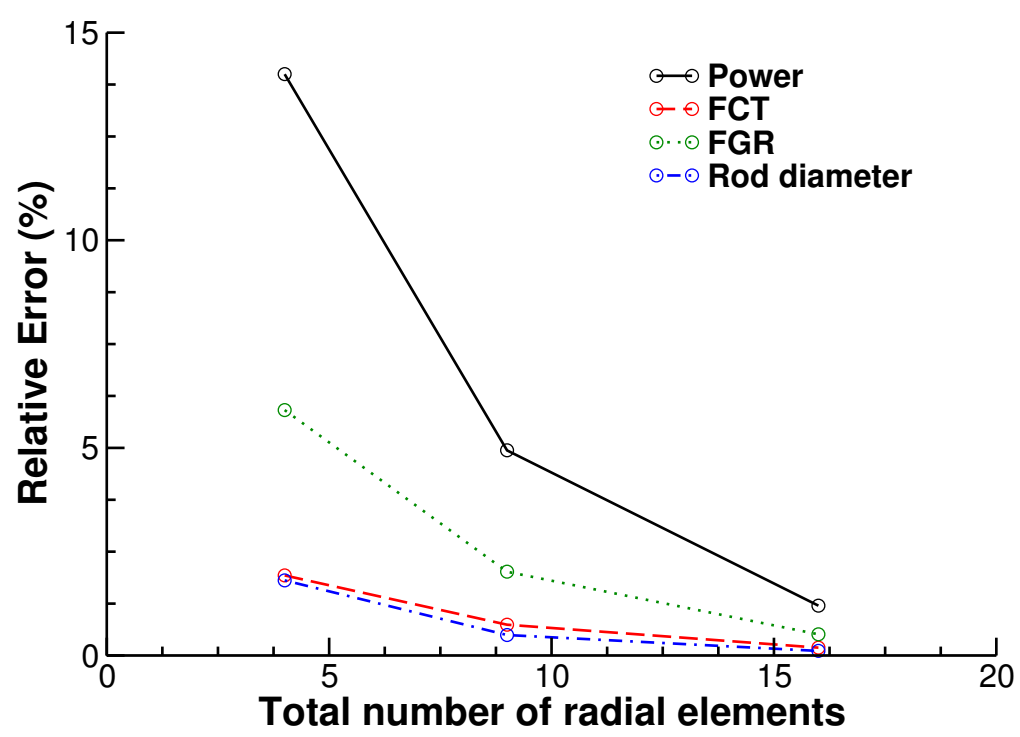

Figure 3.1: Spatial resolution results for the solution verification study. Relative percent error is presented for four metrics of interest: Power, FCT, FGR, and clad diameter as function of the total number of radial elements in the mesh. The relative error is with respect to the finest mesh considered.

IAEA sponsored FUMEX-III Coordinated Research Project and are priority cases from either FUMEX-II [4] or FUMEX-III [5].

\subsubsection{Thermal Behavior}

Accurate fuel temperature prediction is essential for a fuel performance code as temperature is important for assessing fuel rod performance and safety. Additionally, many other important physical phenomena depend highly on temperature.

Temperature comparisons during the first rise to power are significant as they isolate several important aspects of fuel rod behavior before complexities associated with higher burnups are encountered. Accurate prediction of beginning of life (BOL) fuel centerline temperature requires accurate models for the fuel and cladding thermal conductivity and gap conductance. Figure 3.2 summarizes BOL fuel centerline temperature comparisons for the set of experiments in Table 3.1 where such data are available. Plotted is the predicted versus measured fuel centerline temperature as the rod power is increased during power-up. For all cases considered to date, deviations between BISON predictions and experimental data are less than $\pm 10 \%$.

Temperature comparisons during long irradiations show BISON's ability to account for changing fuel thermal conductivity and fuel-clad gap behavior. Comparisons in the paper are given in terms of four different burnup increments: $0 \leq B u<20,20 \leq B u<40,40 \leq B u<60$, and $B u \geq 60 \mathrm{MWd} / \mathrm{kgUO}_{2}$. As an example, temperature comparisons for the interval $20 \leq B u<40$ are shown in Figure 3.3. Although there is slightly more scatter than for the beginning of life comparisons in Figure 3.2, comparisons at higher burnup are very reasonable. 
Table 3.1: Overview of the main integral experimental data used for validation of BISON.

\begin{tabular}{|c|c|c|c|c|c|}
\hline Experiment & Rod & FCT & FGR & $\begin{array}{c}\text { Rod } \\
\text { Dia }\end{array}$ & Ref \\
\hline IFA-431 & $1,2,3$ & $\mathrm{X}$ & & & 15 \\
\hline IFA-432 & $1,2,3$ & $\mathrm{X}$ & & & 15. 16 \\
\hline IFA-515.10 & A1 & $\mathrm{X}$ & & & 17 \\
\hline IFA-597.3 & 8 & $\mathrm{X}$ & $\mathrm{X}$ & & 16 \\
\hline Ris $\varnothing-3$ & AN3,AN4 & $\mathrm{X}$ & $\mathrm{X}$ & & 16 \\
\hline Ris $\emptyset-3$ & AN2 & & $\mathrm{X}$ & $\mathrm{X}$ & 16 \\
\hline Ris $\varnothing-3$ & GE7 & & $\mathrm{X}$ & $\mathrm{X}$ & 16 \\
\hline Ris $\emptyset-3$ & II3 & $\mathrm{X}$ & $\mathrm{X}$ & $\mathrm{X}$ & 16 \\
\hline $\operatorname{Ris} \emptyset-2$ & GE-m & & $\mathrm{X}$ & $\mathrm{X}$ & 16 \\
\hline OSIRIS & J12 & & $\mathrm{X}$ & $\mathrm{X}$ & 16 \\
\hline REGATE & & & $\mathrm{X}$ & $\mathrm{X}$ & 16 \\
\hline USPWR 16x16 & TSQ002,TSQ022 & & $\mathrm{X}$ & $\mathrm{X}$ & 16 \\
\hline IFA-431 (3D) & 4 & $\mathrm{X}$ & & & 15. 18 \\
\hline R.E. Ginna & 2,4 & & $\mathrm{X}$ & $\mathrm{X}$ & 16 \\
\hline OSIRIS & H09 & & $\mathrm{X}$ & $\mathrm{X}$ & 16 \\
\hline HBEP & BK363,BK365 & & $\mathrm{X}$ & & 4 \\
\hline IFA-534 & 18,19 & & $\mathrm{X}$ & & 16 \\
\hline IFA-535 & 809,810 & & $\mathrm{X}$ & & 16 \\
\hline IFA-562.2 & $15,16,17$ & $\mathrm{X}$ & $\mathrm{X}$ & & 19 \\
\hline Ris $\varnothing-3$ & II5 & $\mathrm{X}$ & $\mathrm{X}$ & $\mathrm{X}$ & 16 \\
\hline Tribulation & $\mathrm{BN} 1 / 3, \mathrm{BN} 1 / 4, \mathrm{BN} 3 / 15$ & & $\mathrm{X}$ & $\mathrm{X}$ & 16 \\
\hline
\end{tabular}

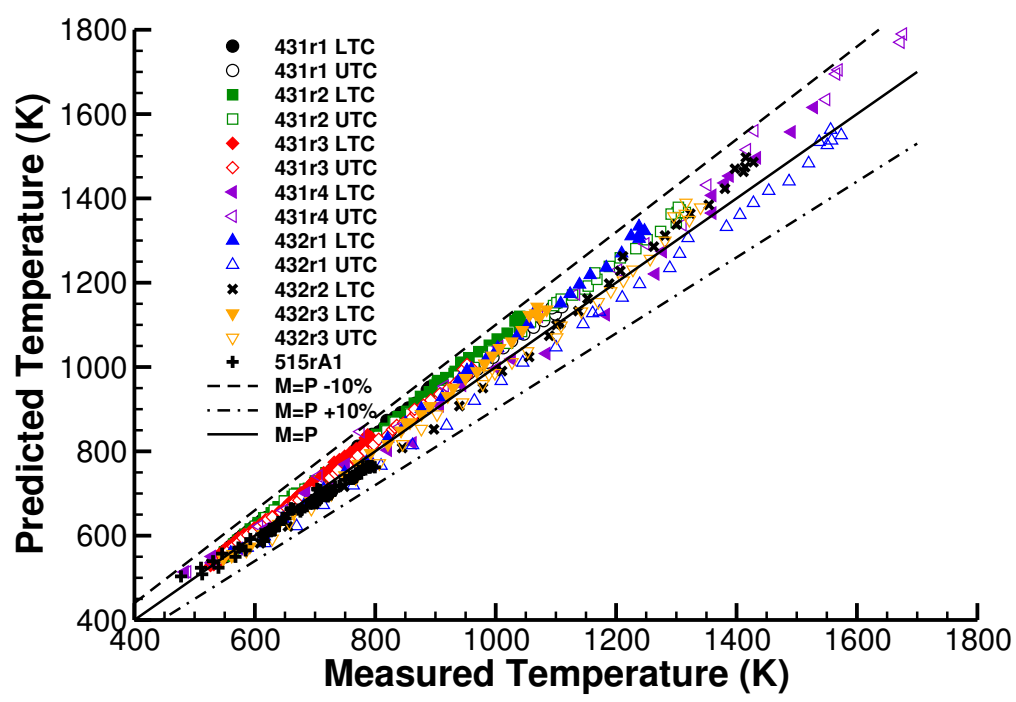

Figure 3.2: BOL measured vs. predicted fuel centerline temperature for fuel rods in IFA-431, IFA-432, and IFA-515.10. LTC and UTC stand for lower and upper thermocouple measurements, respectively.

Five of the experiments listed in Table 3.1 (Risø-3 rods AN3, AN4, II3, II5 and IFA-597.3 rod 8 ) include measurements of fuel centerline temperature during power ramps following base 


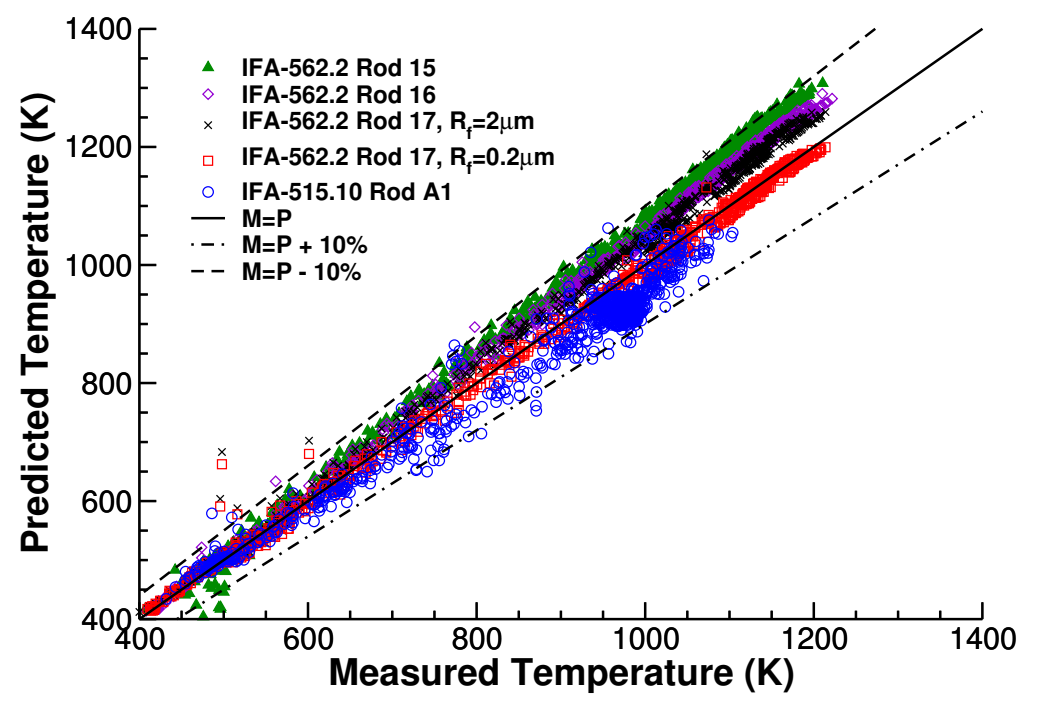

Figure 3.3: Comparison of the measured vs. predicted fuel centerline temperature for through life rods for the burnup range: $20 \leq B u<40$. The $\mathrm{R}_{f}$ parameter in the IFA-562.2 Rod 17 series labels indicate the fuel roughness used in the simulation.

irradiation. These experiments include rods with burnups ranging from 14.5 to $61.6 \mathrm{MWd} / \mathrm{kgU}$. In all cases, experimental rods were refabricated for installation in the test reactor by shortening mother rods. Similar to Figs. 3.2 and 3.3, Fig. 3.4 compares measured and predicted fuel centerline temperatures for the five ramp test experiments. Comparisons are reasonable, however, in contrast to the prior comparisons, some points fall outside the $\pm 10 \%$ difference bands. Certainly approximations involved in simulating the rod refabrication process add uncertainty to the temperature predictions.

\subsubsection{Fission Gas Release}

BISON predictions of integral fuel rod FGR from simulations of the validation cases listed in Table 3.1 have been compared to the available experimental data. Due to the inherent uncertainties of fission gas behavior modeling, a deviation of fuel performance code predictions from the experimental data within a factor of about 2 up and down is generally regarded as satisfactory [4, 5, 20, 21]. A recent uncertainty evaluation study supported the estimation of a factor of 2 as range of tolerance for high calculated FGR values, yet pointing out that higher deviations may be expected for calculated FGR around 10\% and lower [22]. The comparison of BISON calculations and experimental data of integral FGR at the fuel rod end-of-life (EOL) is summarized in Figure 3.5. BISON accuracy in predicting FGR appears to be consistent with state-of-the-art modeling [4, 5, 20] and with the involved uncertainties.

\subsubsection{Rod Diameter}

Accurate simulation of the mechanical behavior of fuel rods is important when attempting to make predictions about cladding structural integrity, for example as a result of pellet cladding 


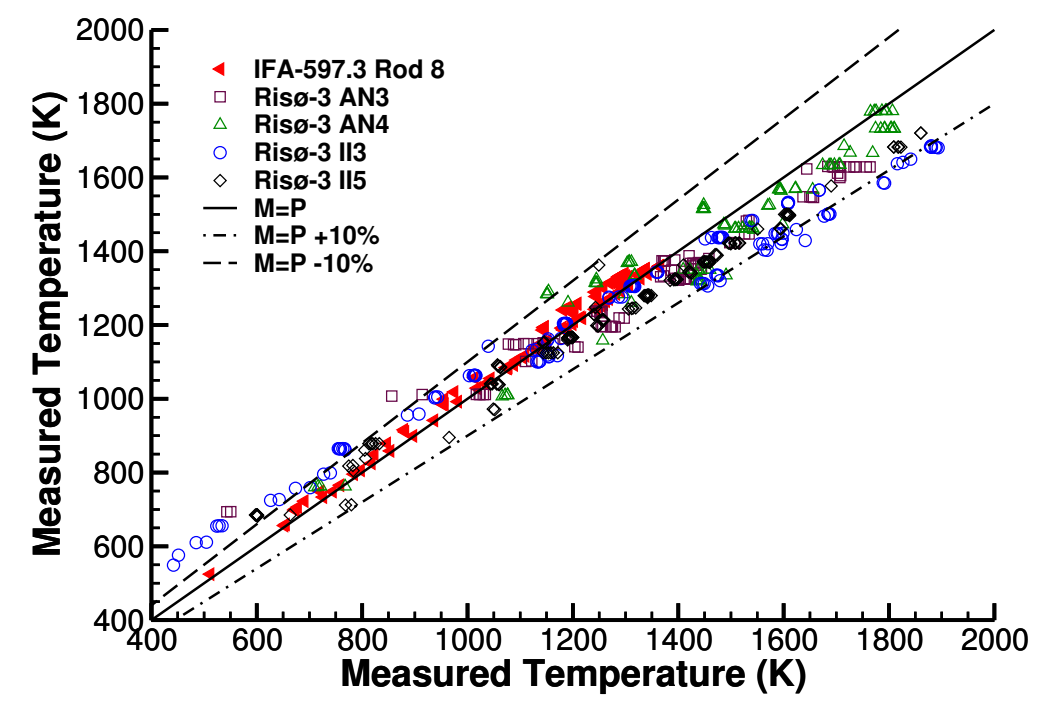

Figure 3.4: Comparison of the measured vs. predicted fuel centerline temperature for fuel rods that experienced power ramps.

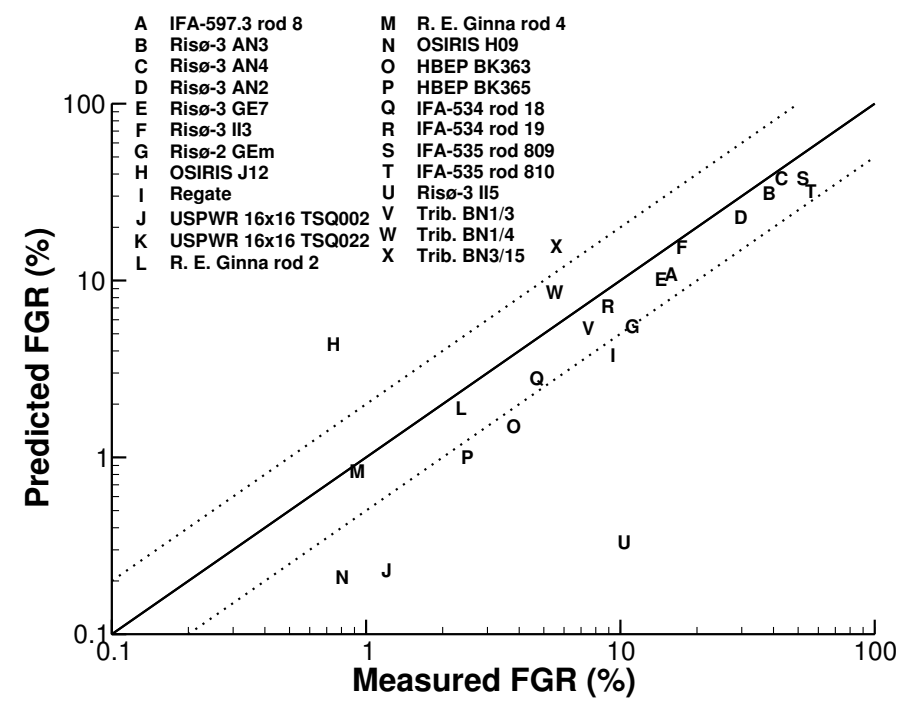

Figure 3.5: Fission gas release measured versus predicted comparisons with a factor of 2 error bands.

mechanical interaction (PCMI). Eleven of the experiments listed in Table 3.1 included rod diameter measurements. All were made post irradiation in a hot cell, with a few including measurements following both base irradiation and after a power ramp.

Table 3.2 summarizes the rod diameter comparisons following base irradiation, in terms of the difference between the measured and predicted values. As indicated in the table, comparisons are made either at the rod axial midplane or averaged over the rod length, based on the available experimental data. The comparisons are separated by cladding type (Zircaloy 4 and Zircaloy 2) 
and ordered according to the final burnup. The comparison results of Table 3.1 are plotted in Figure 3.6 in terms of diameter change vs burnup. In general, the results indicate a tendancy to underpredict the diameter change early in life, and more significantly overpredict the diameter change late in life. The early life comparisons typically occur before fuel-clad contact, when clad deformation is dominated by clad creep down; comparisons thus indicate a tendancy to overpredict clad creep rates. Although the number of low burnup points are limited, comparisons thus far indicate this overprediction is more severe for Zircaloy-2 than for Zircaloy-4. At higher burnups, following fuel-clad contact, the clad diameter increase is controlled by the mechanical behavior of the fuel. The fact that the clad diameter change (measured - predicted) is significantly negative indicates a strong tendancy to overpredict the fuel radial growth.

Table 3.2: Base irradiation

\begin{tabular}{|c|c|c|c|c|}
\hline Case Description & $\begin{array}{l}\text { Clad } \\
\text { Type }\end{array}$ & $\begin{array}{c}\text { Axial } \\
\text { Comparison } \\
\text { Location }\end{array}$ & $\begin{array}{l}\text { Rod Average } \\
\text { Burnup } \\
(\mathrm{MWd} / \mathrm{kgU})\end{array}$ & $\begin{array}{c}\text { Diameter Change } \\
\text { (Meas. - Pred.) } \\
(\mu \mathrm{m})\end{array}$ \\
\hline Tribulation BN1/4 & Zry-4 & Midplane & 19.7 & 16.4 \\
\hline Tribulation BN1/3 & Zry-4 & Midplane & 20.2 & 7.1 \\
\hline OSIRIS J12 & Zry-4 & Average & 23.9 & -18.1 \\
\hline Ris $\varnothing-3$ AN2 & Zry-4 & Average & 31.4 & -16.6 \\
\hline Tribulation BN3/15 & Zry-4 & Midplane & 37.7 & -71.1 \\
\hline OSIRIS H09 & Zry-4 & Average & 46.1 & -42.9 \\
\hline REGATE & Zry-4 & Average & 47.0 & 6.5 \\
\hline Tribulation BN1/4 & Zry-4 & Midplane & 50.6 & -53.2 \\
\hline Tribulation BN1/3 & Zry-4 & Midplane & 50.7 & -70.8 \\
\hline Tribulation BN3/15 & Zry-4 & Midplane & 51.1 & -84.8 \\
\hline R. E. Ginna Rod 2 & Zry-4 & Average & 51.2 & -71.8 \\
\hline R. E. Ginna Rod 4 & Zry-4 & Average & 51.2 & -69.2 \\
\hline USPWR TSQ002 & Zry-4 & Average & 53.2 & -26.3 \\
\hline USPWR TSQ022 & Zry-4 & Average & 58.1 & -40.4 \\
\hline Ris $\emptyset-2$ GE-m & Zry-2 & Average & 15.5 & 35.8 \\
\hline Ris $\varnothing-3$ II3 & Zry-2 & Average & 16.4 & 32.8 \\
\hline Ris $\varnothing-3$ GE7 & Zry-2 & Average & 31.4 & 34.1 \\
\hline Ris $\emptyset-3$ II5 & Zry-2 & Midplane & 39.0 & -78.8 \\
\hline
\end{tabular}

\subsubsection{Summary}

BISON predictions have been compared to a wide variety of LWR experiments. Results indicate:

- Temperature comparisons at begininning of life, during irradiation to high burnup, and during power ramps, are all very reasonable.

- Accuracy in predicting FGR appears to be consistent with state-of-the-art modeling and with the involved uncertainties.

- Comparison of rod diameter indicate a tendancy to underpredict diameter change early in life, and more significantly overpredict diameter change late in life. The comparisons are not satisfactory and require more indepth analyis.

Results from this validation study are being used to define priorities for ongoing code development activities. 


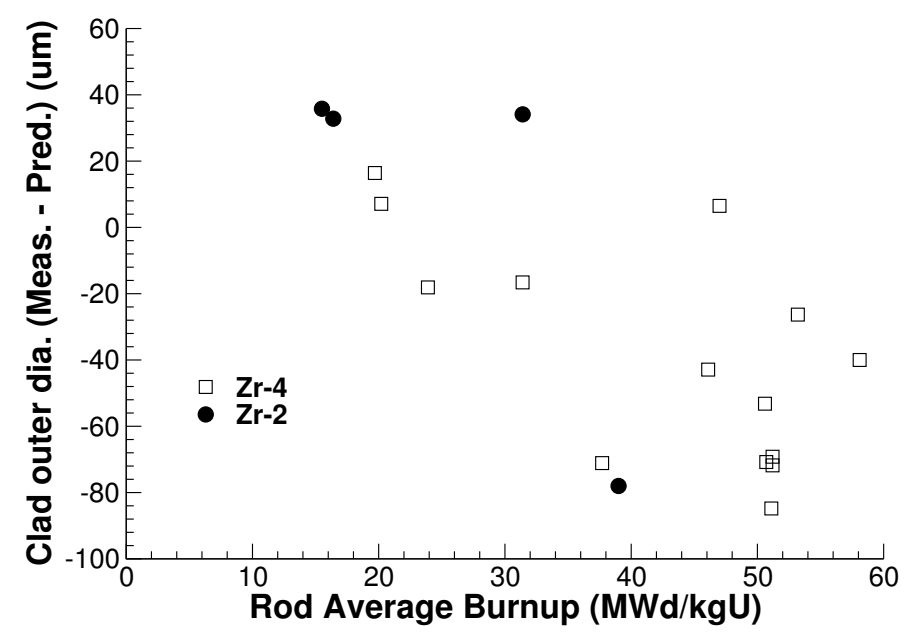

Figure 3.6: The difference between measured and predicted cladding outer diameter as a function of burnup.

\subsection{Multiphysics Coupling Article}

In 2013, we presented a paper [23] at the SMiRT conference regarding coupling approaches for multi-physics. Based on that paper, we received an invitation to write a full paper from Nuclear Engineering and Design. The following is a summary of that paper [24], which was published in August 2015.

The primary concern in fuel performance simulations codes like BISON is solving for temperature (heat equation) and displacements (solid mechanics equations). This is especially challenging given the gap between the fuel and cladding, which represents strong coupling between temperature and displacement. In BISON, and all MOOSE applications, we have options on how to solve these equations in terms of how strongly we couple them. We investigated two approaches, loose and tight coupling.

In loose coupling, the individual physics (temperature from the heat equation and displacement from the solid mechanics equations) in a coupled problem are solved individually, keeping the solutions for the other physics fixed. After a solution is obtained for an individual physics, it is transferred to other physics that depend on it, and solutions are obtained for those physics.

These fixed-point iterations are repeated until convergence is obtained. If there is not a strong two-way feedback between the physics involved, convergence can be obtained quickly with a minimal number of loose-coupling iterations. An advantage of this approach is that it allows for independent codes to be coupled with relatively minor modifications to those codes, and they can each use their own solution strategies that are tailored for their solution domain. The disadvantage of loose coupling is that if there is strong two-way feedback between the physics, that approach can have an unacceptably slow convergence rate and may encounter convergence difficulty.

In tight coupling solution methods, a single system of equations is assembled and solved for the full set of coupled physics. The nonlinear iterations operate on the full system of equa- 
tions simultaneously, taking into account the interactions between the equations for the coupled physics in each iteration. In cases where there is strong coupling between the physics, this approach can have faster convergence rates than loose coupling. The primary disadvantage of this approach is that it necessitates tighter coordination between the codes to solve the individual physics.

The paper described the solution environment used to enable tight and loosely coupled simulations of thermomechanical problems, provided a review of the equations governing thermal and mechanical response, and demonstrated the performance of loose and tight coupling strategies on simple thermomechanical problems with varying degrees of feedback between the two systems. Following these simple demonstrations, the performance of these solution strategies was demonstrated on real-world nuclear engineering problems, first on a simulation of reactor pressure vessel response during pressurized thermal shock conditions and then on a fuel performance simulation. For this summary, simulations for one simple demonstration problem and a simple LWR fuel problem are provided.

\subsubsection{Thermomechanical contact example}

The simplified demonstration is a thermomechanics problem with thermal and mechanical contact between two domains. This problem was developed to compare the performance of the coupling approaches when applied to a difficult coupled thermal and mechanical contact problem with an evolving gap between two domains. Each domain is a rectangular block. One block is situated at a distance of 0.25 above and offset length-wise by 1.25 from the other block. The lower half of the top block is assigned a coefficient of thermal expansion of $50 \times 10^{-6}$. In other words, the lower half of the top block should have 5 times the thermal strain when compared to all other points in the domain given an equal change in temperature. These problems were run using one processor. The unitless geometry and material properties are for demonstration purposes and are not representative of a real problem.

The upper block is cantilevered at the far end while the bottom surface of the lower block is fixed from translation in any direction. The simplified gap conductance model was used with a thermal conductivity of $4 \times 10^{-2}$, and a minimum gap size of $5 \times 10^{-4}$.

Each block has an initial temperature of 100. The temperature of the top surface of the upper block is held constant at 100 for the entire simulation. The temperature of the bottom surface of the lower block is increased from 100 to 1000 during the first 8 quasi-static load steps of this simulation, held at 1000 through load step 16 then decreased to 100 from load step 16 to load step 24, which is the final load step. All other surfaces are thermally insulated. The right side of the top block is initially held fixed, and is then monotonically displaced downward toward the bottom block (thereby closing the gap completely), from load step 8 to load step 16, at which point the displacement on that boundary is -0.26 .

As the temperature of the lower block increases, heat is transferred to the upper block via the gap, which causes the upper block to thermally expand. The higher coefficient of thermal expansion in the lower half of the upper block causes the block to bend away from the lower block. After the lower block reaches a temperature of 1000, the upper block is lowered, so that the gap decreases, causing increased heat transfer from the lower block to the upper block, which results in more bending. After the upper block makes contact with the lower block, the 
temperature of the lower block is decreased, which reverses the bending of the upper beam, and thus decreases the gap size. As for the single-block problem, these cases were all run on a single processor using a direct solver for preconditioning.

Temperature contour plots and the displaced mesh are shown in Figure 3.7 at load steps 0, 8, 16 , and 24. The results for the primary solution variables (displacements and temperatures) for

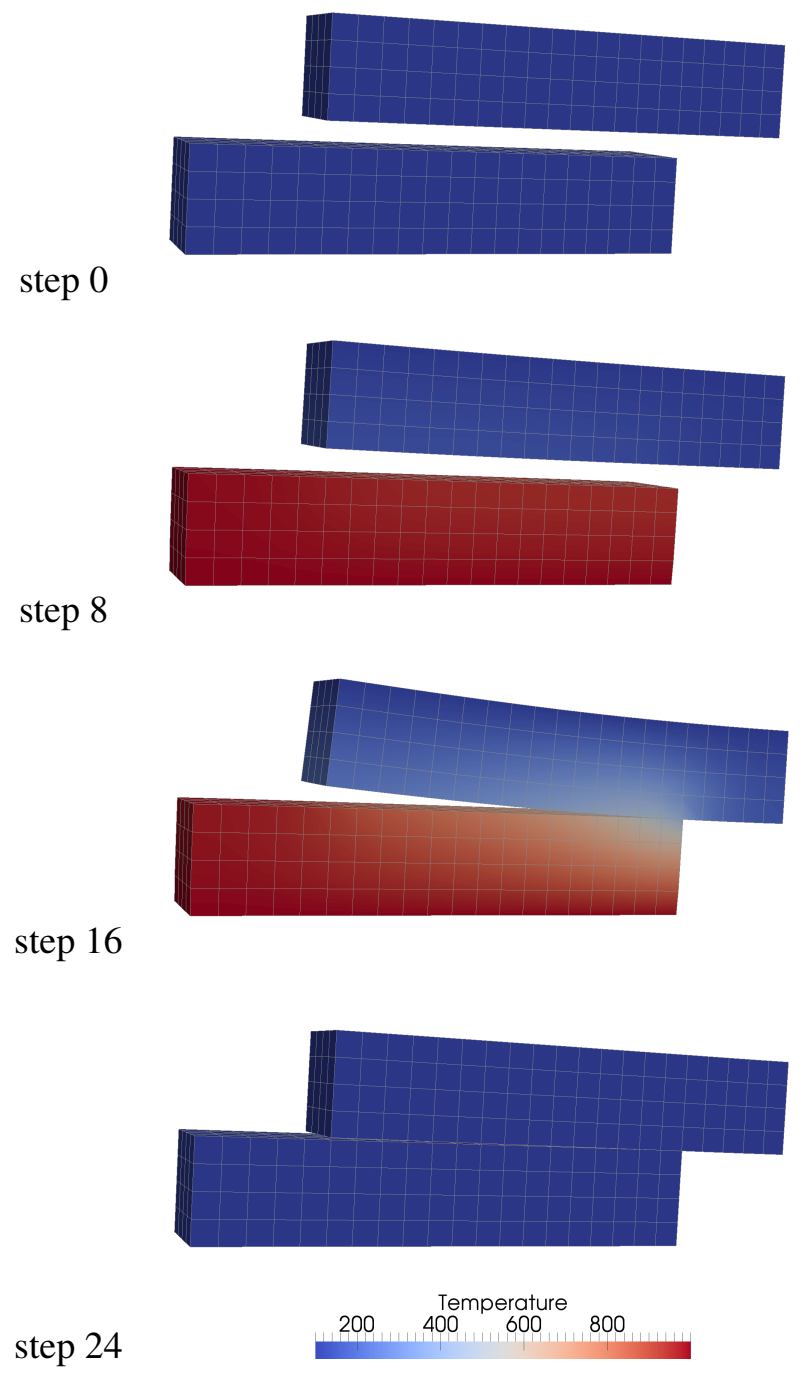

Figure 3.7: Thermomechanics simulation with gap at several steps.

the tightly and loosely coupled simulations were identical within the convergence limits.

Figure 3.8 shows a comparison of iteration counts for each step for the tight and loose coupling approaches for this model, as well as these same iteration counts plotted cumulatively. It shows the nonlinear iterations taken in the tight coupling approach, the number of fixed-point iterations in the loose coupling approach, and the total number of nonlinear iterations taken by the individual physics solutions in the loose coupling approach, accumulated for all fixed-point 
iterations in each step.

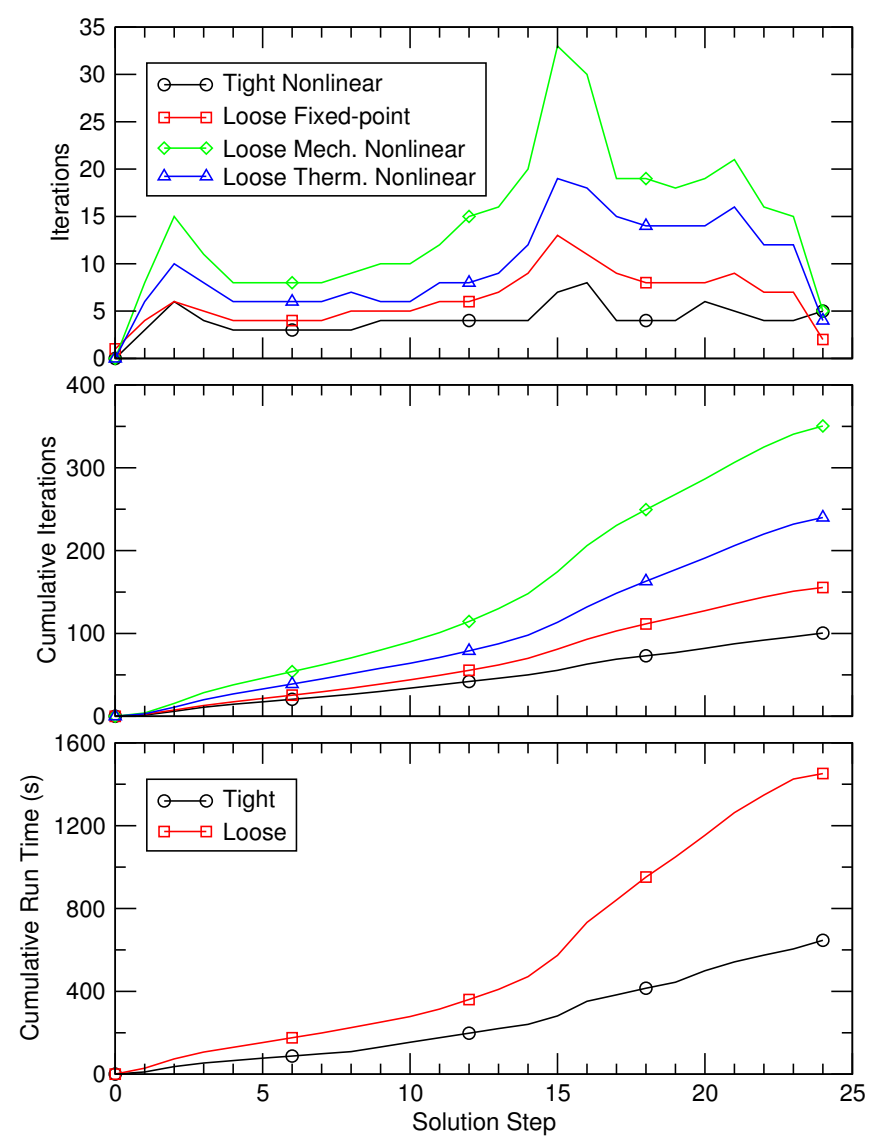

Figure 3.8: Comparison of solver performance for loose and tight coupling approaches for beam model. (top) Iterations per step (middle) Cumulative iterations up to current step (bottom) Cumulative wall clock run time.

These plots of iteration counts show that the tightly coupled solution took roughly five nonlinear iterations for each step, except at the steps when mechanical contact was first being established. The loose coupling case took about five fixed-point iterations per step early in the analysis, when the gap was large, but this number increased as the beams came into contact. The loose coupling approach overall took roughly twice as long as the tight coupling approach. This increased computational cost is largely due to the increased cost when the beams were in contact, as evident by the increased slope of the cumulative run time plot for the loosely coupled approach. The slope of the cumulative run time plot is more constant for the tight coupling approach.

\subsubsection{LWR fuel performance simulation example}

Tightly and loosely coupled strategies are evaluated using a 2D axisymmetric simulation of a short section of an LWR fuel pin. The details of this model are documented in [1]. The assumed 
geometry, shown in Figure 3.9, includes ten individual $\mathrm{UO}_{2}$ pellets, $\mathrm{Zr}-4$ cladding, an initial 80 $\mu \mathrm{m}$ pellet-clad gap, and an open region to simulate the upper plenum. The clad outer surface temperature is held fixed at $605 \mathrm{~K}$. The rod power is assumed to rise linearly over three hours and then held constant for $8 \times 10^{7} \mathrm{~s}$ (about 30 months). An axially varying power profile is applied over the length of the fuel column. Although this variation is unrealistic for a short rodlet, the resulting axial variation in fuel temperature ensures that once pellet-clad contact begins, some portion of the rodlet is coming into contact over the remaining irradiation period. The finite element mesh for a single fuel pellet and neighboring clad is also shown in Figure 3.9. This mesh has 2448 linear quadrilateral elements and 2898 nodes.

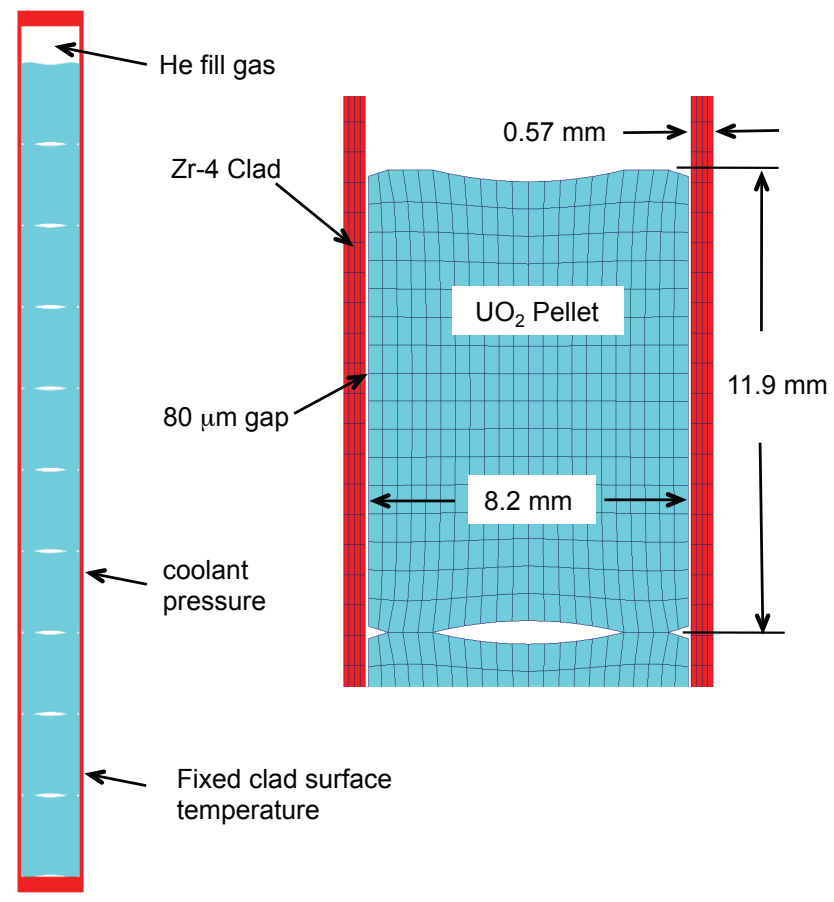

Figure 3.9: Geometry, materials and mesh used to simulate an axisymmetric discrete-pellet fuel rodlet.

The fuel performance simulation results obtained from the tightly and loosely coupled solution strategies are effectively identical. Figure 3.10 shows representative temperature contour plots at the final time step. The axial variation in the temperature is reflective of the nonuniform axial profile applied to this model. At this point in time, sufficient fuel swelling and cladding creep have occurred to completely close the gap between the fuel and cladding.

Figure 3.11 shows a comparison of iteration counts for each step for the tight and loose coupling approaches. Early in the analysis, the loose coupling approach required a small number of fixed-point iterations, but as the gap between the fuel and cladding closed, the number of fixed-point iterations required per step increases considerably. The tightly coupled solution, on the other hand, required a fairly constant number of nonlinear iterations per step. Later in the analysis, after the gap completely closed, the number of fixed-point iterations decreased for the 


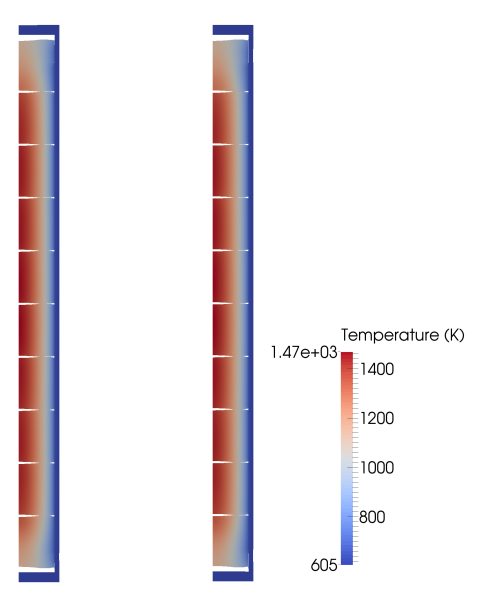

(a)

(b)

Figure 3.10: Contour plot of temperature at the end of simulation. (a) Tightly coupled (b) Loosely coupled. Coordinates are scaled by 0.5 in the axial direction.

loose coupling case. The overall run time for the loose coupling case was significantly higher than for the tight coupling case.

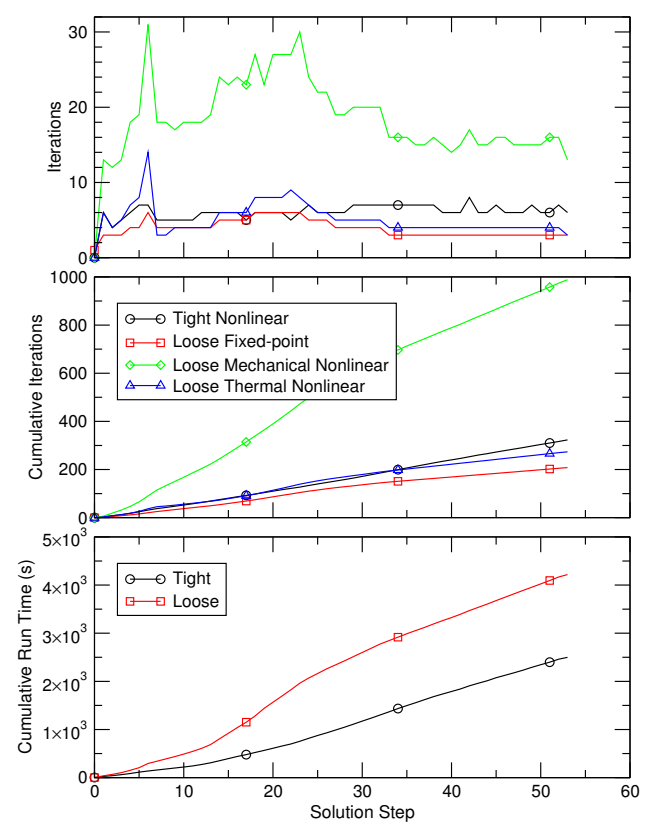

Figure 3.11: Comparison of solver performance for loose and tight coupling approaches for fuel model. (top) Iterations per step (middle) Cumulative iterations up to current step (bottom) Cumulative wall clock run time. 


\subsubsection{Conclusions}

- A loose coupling strategy that uses a series of fixed-point iterations to repeatedly solve the individual physics models and transfer results between them generates results that are effectively identical to those obtained from a tightly coupled solution of the full coupled system of equations. This has been demonstrated on a variety of thermomechanical problems. For an objective comparison of the two approaches, care must be taken to use consistent convergence criteria.

- For problems that involve essentially one-way feedback between the thermal and mechanical response, a small number of fixed-point iterations are required for convergence in the loose coupling approach, and loose coupling gives better overall performance than tight coupling. In the models of this class studied here, overall run times for tight coupling were about $30 \%$ to $60 \%$ longer than those for loose coupling. This stands to reason, as it is more efficient to solve two smaller systems of equations than one large system of equations.

- As the amount of two-way feedback between the thermal and mechanical response increases, loose coupling requires an increasing number of fixed-point iterations, while the number of nonlinear iterations for a tightly coupled solution of the full system remains relatively constant. In the models studied here with strong two-way feedback, the run times for loose coupling ranged from about $70 \%$ to $275 \%$ longer than those for tight coupling.

- Two phenomena observed here that can lead to strong two-way feedback between the thermal and mechanical solutions are incremental thermal gradients and thermal contact. In both cases, a change in the configuration due to the mechanical response leads to a change in the thermal solution. This effect is typically much more pronounced for thermal contact than for incremental thermal gradients.

- The fuel performance simulation considered here was much better solved using a tightly coupled approach.

\subsection{Reactivity Insertion Accident Analysis}

Recent BISON development efforts have focused on code extensions to enable analysis of accident behavior, both LOCAs and Reactivity Insertion Accidents (RIAs). Validation of BISON for LOCA and RIA behavior is also a priority. Late in FY-15 the BISON development team learned of a new international OECD benchmark activity designed to investigate the ability of transient fuel performance codes to simulate RIA behavior. The INL became involved in this activity and has developed preliminary models of several of the benchmark cases.

As an example of this effort, the model and mesh for Case 3 from the benchmark exercise is shown in Figure 3.12. The geometry is simple, including only a $10 \mathrm{~cm}$ length of $\mathrm{UO}_{2}$ fuel, encased in standard $\mathrm{Zr}-4$ cladding, and filled with helium. The case includes a $50 \mu \mathrm{m}$ gap and assumes free slip between the fuel and clad following contact. The temperature of the clad 
exterior is held at $553 \mathrm{~K}$ with a coolant pressure of $15.5 \mathrm{MPa}$. The input power pulse, which occurs over $60 \mu s$, is shown in Figure 3.13 .

Figure 3.13 also shows the computed temperature histores for Case 3 at the fuel centerline, fuel surface and inner wall of the clad. The fuel centerline temperature rises rapidly in response to the power pulse, and then remains nearly constant over the $0.2 \mathrm{~s}$ time period plotted. The fuel surface temperture rises at a similar rate until it comes into contact with the cladding, occurring approximately $20 \mathrm{~ms}$ after the beginning of the power pulse. As indicated in the figure, contact with the cooler cladding results in a small but temporary drop in the fuel surface temperature. This decrease ends as the clad temperature increases to a point that the fuel outer surface and clad inner surface temperatures have very similar time histories, separated only by an approximately $50 \mathrm{~K}$ temperature drop across the surfaces in contact.

The symbols in the centerline temperature curve of Figure 3.13 indicate individual timesteps, and show the very small time increments needed to capture the rapid RIA transient. Note that the time step algorithms in BISON permit automatic reduction or increase in step size in response to physical behavior in the calculation.

In cooperation with a PhD student (Charles Folsom) working at INL, most of the ten OECD RIA benchmark cases have been completed. Results are currently being compared to a variety of other transient fuel performance codes. A conference paper on this work has been submitted to the PHYSOR meeting in 2016.

\subsection{Uncertainty Analysis in Fuel Modeling}

In 2015, the BISON team participated in Organization for Economic Cooperation and Development (OECD) benchmark program on uncertainty and best estimate modeling described in an OECD report entitled "Benchmark for Uncertainty Analysis in Modelling (UAM) for Design, Operation, and Safety Analysis of LWRs". The BISON effort focused on the cases outlined in Chapter 2 Definition of Exercise II-1: Fuel Modelling. The report outlines the specifications for a variety of numerical and experimental test cases for PWR, BWR, and VVER reactor conditions for both normal operating and transient scenarios. The reason for participation in this OECD benchmark is that there has been an increased demand in providing best estimate predictions to be provided with confidence bounds taking into account model, simulation, and experimental uncertainty. The BISON team's participation this fiscal year was focused on Case 2a from the report which simulated a PWR under normal operating conditions. To perform the uncertainty quantification and sensitivity analyses BISON was coupled to Sandia's National Laboratories' (SNL) Dakota software. This work was completed in collaboration with Laura Swiler and Rod Schmidt from SNL.

The report outlined the nominal conditions and geometry of the fuel rod to be simulated and their associated uncertainties. Table 3.3 outlines the uncertain parameters and the type of distribution. For normal distributions parameter 1 corresponds to the mean value and parameter 2 the standard deviation, whereas for uniform distributions parameter 1 corresponds to the minimum value and parameter 2 the maximum value. In the cases where parameter 1 is equal to 1.0 , the parameter varied was a scaling factor applied to the calculation of the uncertain parameters listed. The first uncertain parameters were core boundary conditions, the next six were 


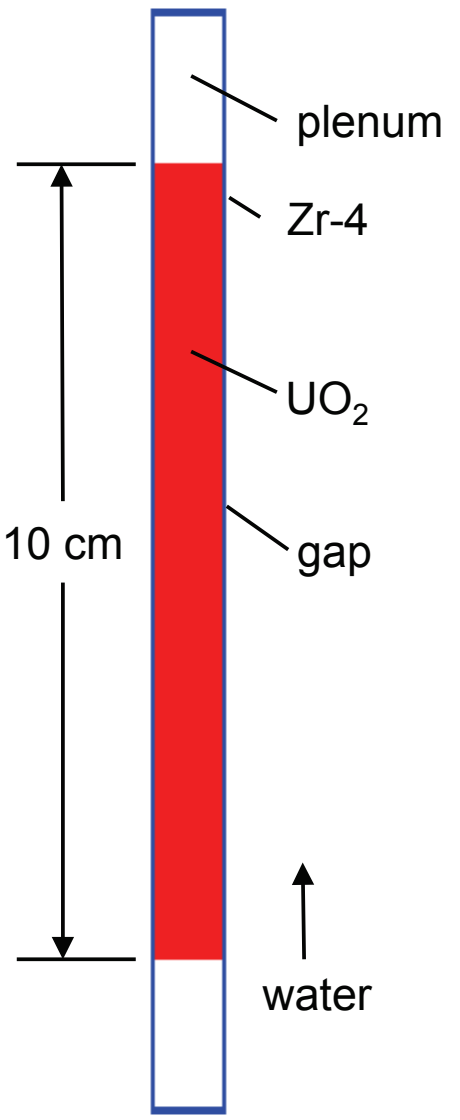

(a)

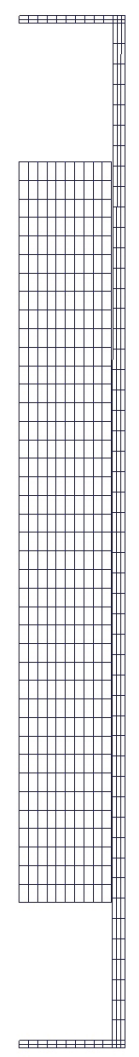

(b)

Figure 3.12: Geometry (a) and 2D axisymmetric mesh (b) for Case 3 of the OECD RIA benchmark exercise. Note that the mesh is magnified $3 \mathrm{x}$ in the radial direction. 


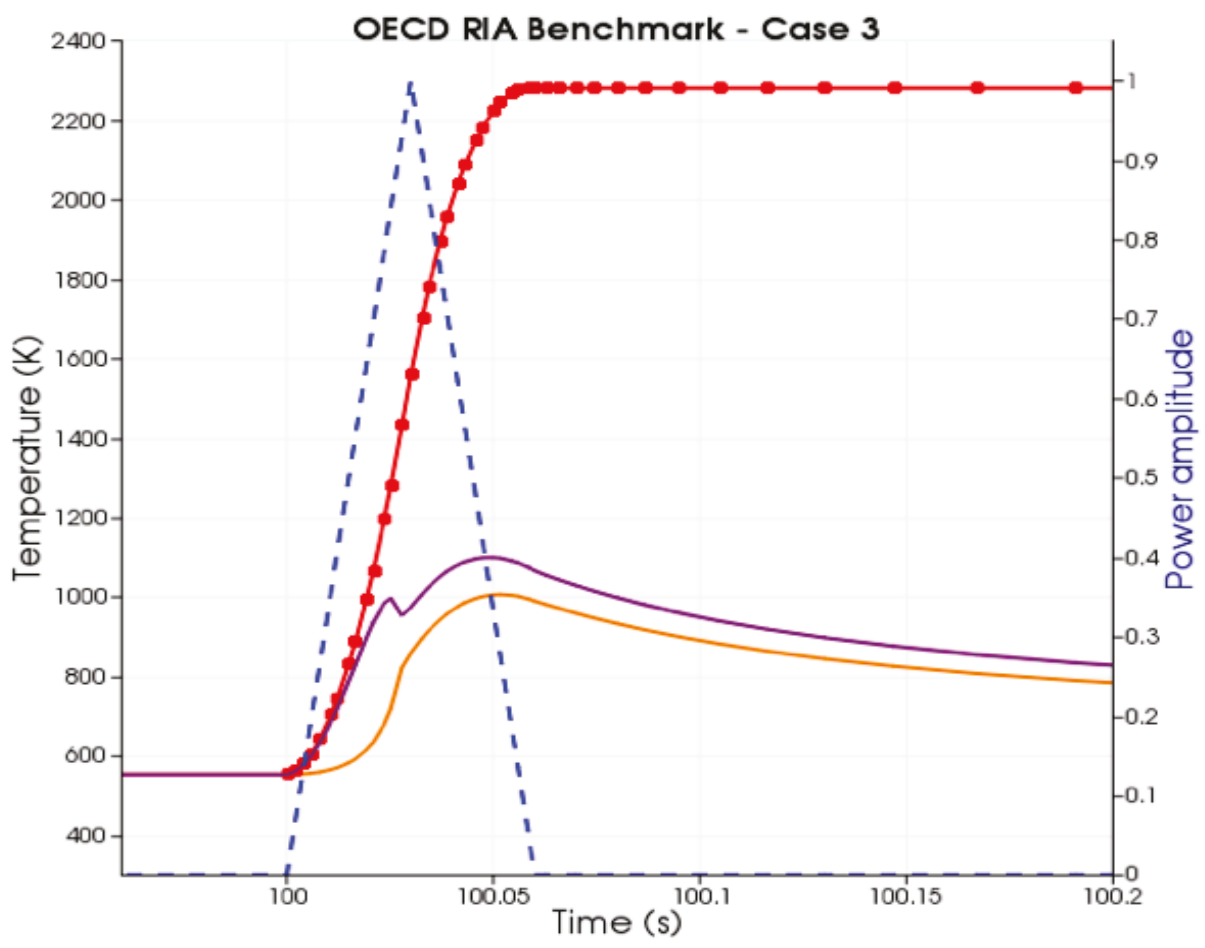

Figure 3.13: Computed temperature histories at the fuel centerline and surface and clad inner wall, for Case 3 of the OECD RIA benchmark exercise. Also show in the input power pulse. 
listed as manufacturing tolerances/geometric uncertainties, and the final seven were user defined uncertainties associated with properties within the fuel, cladding and gap.

Table 3.3: Input distributions used for the OECD case 2a fuels benchmark study.

\begin{tabular}{lccc}
\hline Uncertain Parameter & Type & Parameter 1 & Parameter 2 \\
\hline System Pressure [Pa] & Normal & $1.551 \times 10^{7}$ & 51648.3 \\
Mass Flux [kg/m $\left.{ }^{2}-\mathrm{s}\right]$ & Normal & 3460.0 & 57.67 \\
System Power [W/m] & Normal & 1.0 & 0.016667 \\
Inlet Temperature [K] & Uniform & 558.0 & 564.0 \\
Cladding Thickness [m] & Normal & $6.7 \times 10^{-4}$ & $8.3 \times 10^{-6}$ \\
Cladding Roughness [m] & Normal & $5.0 \times 10^{-7}$ & $1.0 \times 10^{-7}$ \\
Fuel Pellet Radius [m] & Normal & $4.7 \times 10^{-3}$ & $3.335 \times 10^{-6}$ \\
Fuel Density [kg/m $\left.{ }^{3}\right]$ & Normal & 10299.24 & 51.4962 \\
Fuel Pellet Roughness [m] & Normal & $2.0 \times 10^{-6}$ & $1.6667 \times 10^{-7}$ \\
Rod Fill Pressure [Pa] & Normal & $1.2 \times 10^{6}$ & 40000.0 \\
Solid Fuel Swelling $\pm 20 \%[-]$ & Normal & $5.58 \times 10^{-5}$ & $5.577 \times 10^{-6}$ \\
Clad Creep Rate $\pm 30 \%\left[\mathrm{~s}^{-1}\right]$ & Normal & 1.0 & 0.15 \\
Fuel Thermal Conductivity $\pm 10 \%[\mathrm{~W} / \mathrm{m}-\mathrm{K}]$ & Normal & 1.0 & 0.05 \\
Clad Thermal Conductivity $\pm 5 \mathrm{~W} / \mathrm{m}-\mathrm{K}$ & Normal & 16.0 & 2.5 \\
Fuel Thermal Expansion $\pm 15 \%$ & Normal & $1.0 \times 10^{-5}$ & $7.5 \times 10^{-7}$ \\
Gas Conductivity $\pm 5 \%[\mathrm{~W} / \mathrm{m}-\mathrm{K}]$ & Normal & 1.0 & 0.025 \\
Gap Thickness [m] & Normal & $9.0 \times 10^{-6}$ & $8.33 \times 10^{-6}$ \\
\hline
\end{tabular}

The outputs desired included the mean and standard deviation of each of the output metrics, and the Pearson or Spearman correlation coefficients against each of the uncertain input parameters for eight burnup levels, $0,5,10,20,30,40,50$, and $60 \mathrm{GWd} / \mathrm{MTU}$. The output metrics of interest included centerline temperature at 13 axial locations along the rod, maximum fuel centerline temperature, maximum cladding surface temperature, fission gas fraction, cladding creep strain, axial elongation, fuel thermal expansion coefficient, cladding thermal conductivity, cladding thermal expansion coefficient, and gap width. To perform the sensitivity analyses and uncertainty quantification, 300 BISON runs were completed using a Latin Hypercube Sampling study generated in Dakota. The results were compiled in a spreadsheet provided by OECD to allow for consistent comparison of results across various groups and codes.

Figures 3.14 and 3.15 contain scatterplots of the centerline temperature at 3 axial locations (bottom, mid plane, top) along the fuel stack versus the user-defined uncertainties at a burnup level of 5 and $50 \mathrm{GWd} / \mathrm{MTU}$, respectively. The scatterplots provide a way to visualize the sensitivity analysis results. It is observed that the fuel thermal conductivity (column 3) has a strong negative correlation with these three axial locations at both burnup levels. As the thermal conductivity of the fuel increases (a scale factor approaching 1.1) the temperatures decrease as expected. Moreover, one can see that that scattering of the points becomes less indicating a much more negative correlation coefficient at a burnup of $50 \mathrm{GWd} / \mathrm{MTU}$ than at a burnup of 5 $\mathrm{GWd} / \mathrm{MTU}$. These figures are used to provide an example of the type of data that can be obtained through sensitivity analyses. 


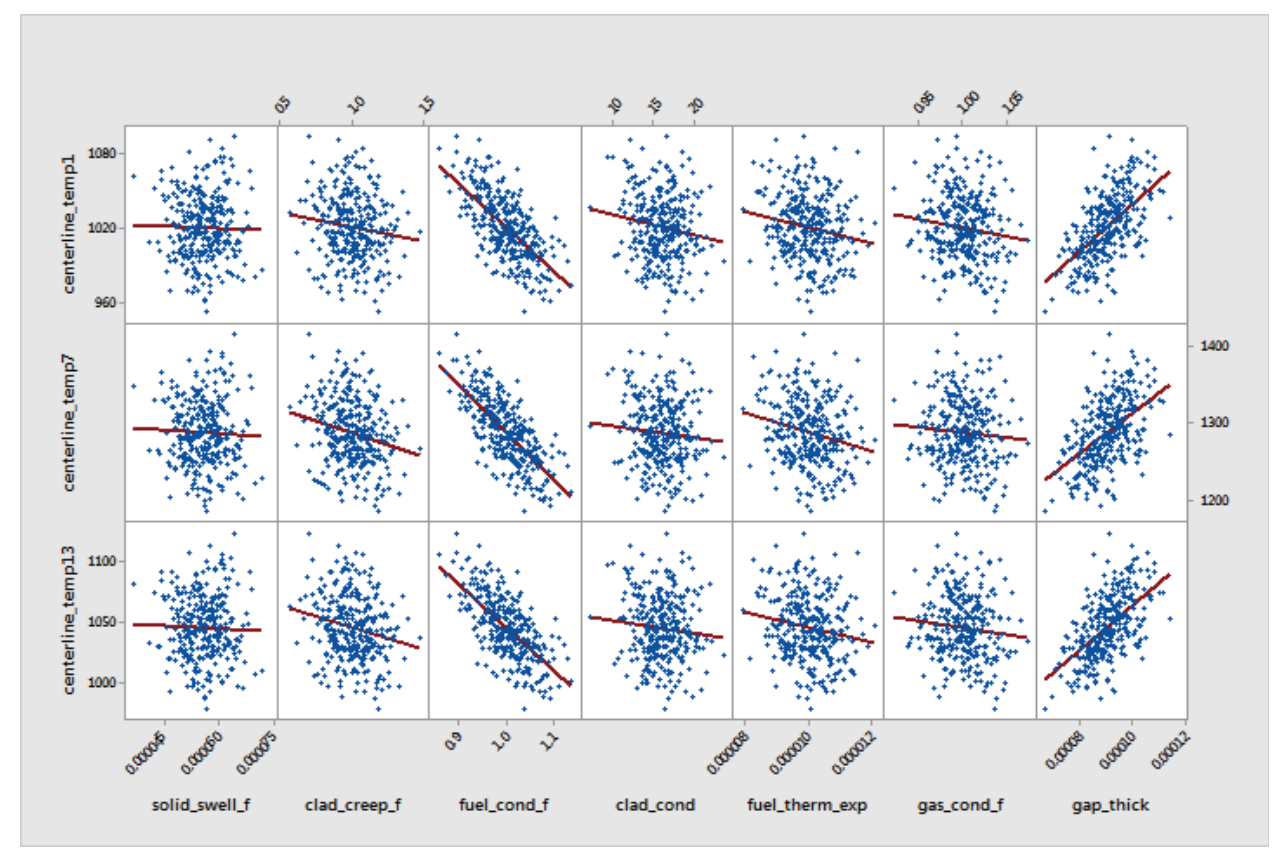

Figure 3.14: Scatterplots of centerline temperature at 3 locations vs. user-defined uncertainties, burnup $=5 \mathrm{GWd} / \mathrm{MTU}$

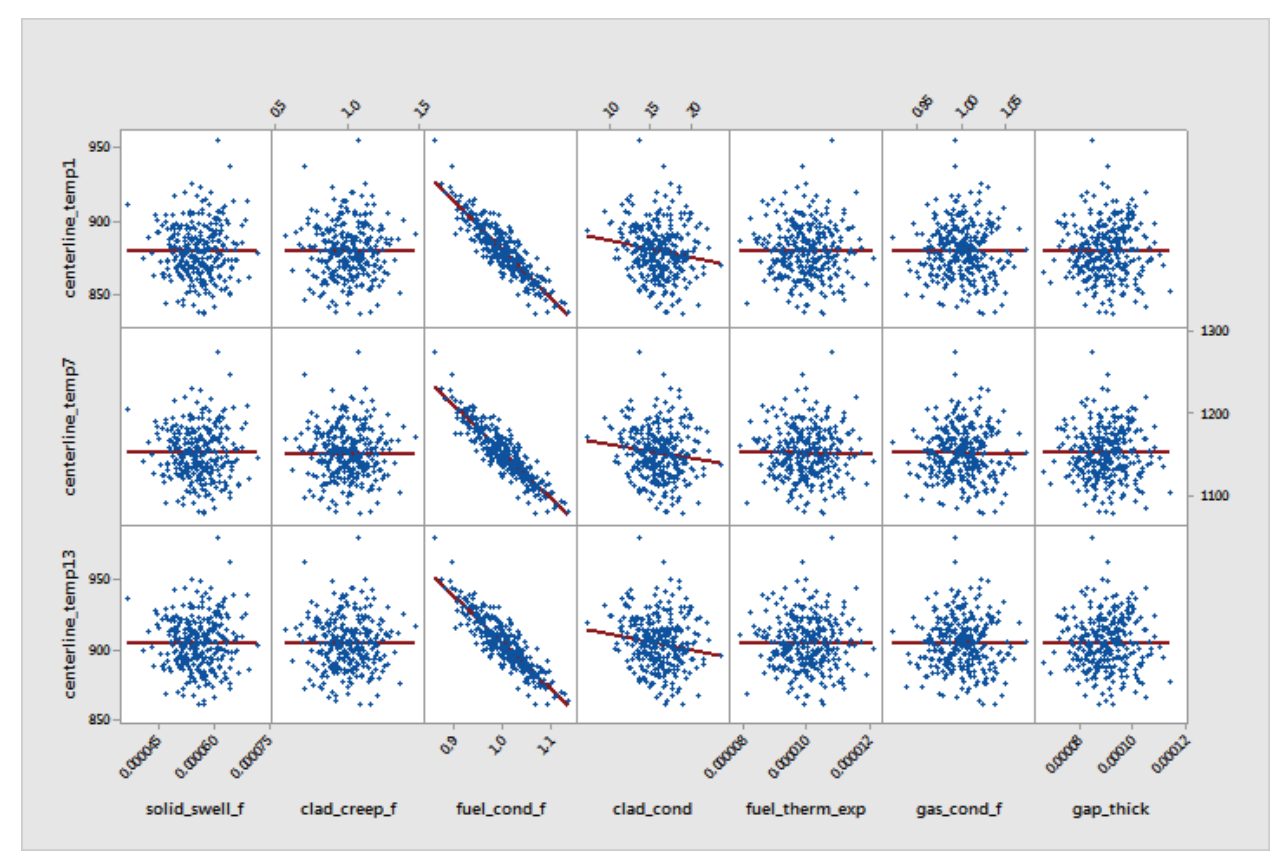

Figure 3.15: Scatterplots of centerline temperature at 3 locations vs. user-defined uncertainties, burnup $=50 \mathrm{GWd} / \mathrm{MTU}$ 


\section{Future Work}

In 2016, the BISON team plans to improve code robustness and efficiency, expand accident simulation capability, continue material model development for fast oxide fuels, improve code documentation and software quality practices, and continue the validation effort.

Of key importance is to improve the robustness and efficiency of the smeared cracking algorithms for oxide fuel. Smeared cracking must be tested and used in concert with fuel creep and stress-based densification models. Work will also continue to unify the solid mechanics and tensor mechanics coding used in BISON.

Accident capabilities will be expanded, specifically to include 1) temperature dependent yield strength and rate dependent plasticity for RIAs and 2) improvement to the low to high temperature creep transition for LOCAs.

Code cleanup and documentation will remain a priority, including improvement to software quality practices.

Validating BISON against experimental measurements is an ongoing effort that will continue in 2016. An increase in full-length fuel rod simulations is planned. Accurately predicting PCMI is a significant concern and will be addressed by consideration of multiple simpler separate effects experiments. The development of automated validation metrics for nightly runs is planned.

The BISON team will provide user support throughout the year via the bison-users@inl.gov group email list, training sessions, and updates to documentation. 


\section{Acknowledgements}

The submitted manuscript has been authored by a contractor of the U.S. Government under Contract DE-AC07-05ID14517. Accordingly, the U.S. Government retains a non-exclusive, royalty free license to publish or reproduce the published form of this contribution, or allow others to do so, for U.S. Government purposes. 


\section{Bibliography}

[1] R. L. Williamson, J. D. Hales, S. R. Novascone, M. R. Tonks, D. R. Gaston, C. J. Permann, D. Andrs, and R. C. Martineau. Multidimensional multiphysics simulation of nuclear fuel behavior. J. Nucl. Mater., 423:149-163, 2012.

[2] J. D. Hales, K. A. Gamble, B. W. Spencer, S. R. Novascone, G. Pastore, W. Liu, D. S. Stafford, R. L. Williamson, D. M. Perez, and R. J. Gardner. BISON users manual. Technical Report INL/MIS-13-30307, Rev. 3, Idaho National Laboratory, September 2015.

[3] J. D. Hales, R. L. Williamson, S. R. Novascone, G. Pastore, B. W. Spencer, D. S. Stafford, K. A. Gamble, D. M. Perez, and W. Liu. BISON theory manual: The equations behind nuclear fuel analysis. Technical Report INL/EXT-13-29930, Rev.2, Idaho National Laboratory, September 2015.

[4] IAEA. Fuel Modelling at Extened Burnup (FUMEX-II): Report of a Coordinated Research Project 2002-2007. Technical Report IAEA-TECDOC-1687, International Atomic Energy Agency, 2002-2007.

[5] IAEA. Improvement of Computer Codes Used for Fuel Behaviour Simulation (FUMEXIII): Report of a Coordinated Research Project 2008-2012. Technical Report IAEATECDOC-1697, International Atomic Energy Agency, 2008-2012.

[6] D. M. Perez, R. L. Williamson, S. R. Novascone, R. J. Gardner, K. A. Gamble, A. T. Rice, G. Pastore, J. D. Hales, and B. W. Spencer. Assessment of BISON: A nuclear fuel performance analysis code. Technical Report INL/MIS-13-30314, Rev. 2, Idaho National Laboratory, September 2015.

[7] M. Inoue, K. Maeda, K. Katsuyama, K. Tanaka, K. Mondo, and M. Hisada. Fuel-tocladding gap evolution and its impact on thermal performance of high burnup fast reactor type uranium-plutonium oxide fuel pins. J. Nucl. Mater., 326(1):59-73, 2004.

[8] Aydin Karahan. Modeling of thermo-mechanical and irradiation behavior of metallic and oxide fuels for sodium fast reactors. PhD thesis, Massachusetts Institute of Technology, June 2009.

[9] P. G. Lucuta, H. J. Matzke, and I. J. Hastings. A pragmatic approach to modelling thermal conductivity of irradiated $\mathrm{UO}_{2}$ fuel: review and recommendations. J. Nucl. Mater, 232:166-180, 1996.

[10] J. L. Routbort. Compressive creep of mixed oxide fuel pellets. J. Nucl. Mater., 44:247-259, 1972. 
[11] M. E. Cunningham, C. E. Beyer, P.G. Medvedev, and G. A. Berna. FRAPTRAN: A Computer Code for the Transient Analysis of Oxide Fuel Rods. Technical Report NUREG/CR6739 Vol.1, PNNL-13576.

[12] F. F. Cadek, D. P. Dominicis, H. C. Yeh, and R. H. Leyse. Pwr flecht final report supplement. Technical Report WCAP-7931, October 1972.

[13] J. K. Fink and L. Leibowitz. Thermodynamic and transport properties of sodium liquid and vapor. Technical Report ANL/Re-95/2, ANL Reactor Engineering Division, 1995.

[14] J. D. Hales, S. R. Novascone, B. W. Spencer, R. L. Williamson, G. Pastore, and D. M. Perez. Verification of the BISON fuel performance code. Ann. Nuclear Energy, 71:81-90, September 2014.

[15] C. R. Hann, D. D. Lanning, E. R. Bradley, R. K. Marshall, M. E. Cunningham, and R. E. Williford. Data Report for the NRC/PNL Halden Assembly IFA-432. Technical Report NUREG/CR-0560, PNL-2673, 1978.

[16] E. Sartori, J. Killeen, and J. A. Turnbull. International Fuel Performance Experiments (IFPE) Database. OECD-NEA, 2010, available at http://www.oecdnea.org/science/fuel/ifpelst.html.

[17] T. Tverberg, M. Amaya. Study of thermal behavious of $\mathrm{UO}_{2}$ and (U,Gd)O $\mathrm{O}_{2}$ to high burnup (IFA-515). Technical Report HWR-671, Halden, February 2001.

[18] J. D. Hales, D. M. Perez, R. L. Williamson, S. R. Novascone, B. W. Spencer, and R. C. Martineau. Validation of the BISON 3D fuel performance code: Temperature comparisons for concentrically and eccentrically located fuel pellets. In Enlarged Halden Programme Group Meeting: Proceedings of the Fuels and Materials Sessions, volume HPR378, Storefjell Resort Hotel, Norway, March 10-15 2013. OECD Halden Reactor Project.

[19] P. Lösönen. Early-in-life Irradiation of IFA-562.2 (The Ultra High Burn-up Experiment. Technical Report HWR-247, OECD Halden Reactor Project, 1989.

[20] Fuel modelling at extended burnup: Report of the Co-ordinated Research Programme on Fuel Modelling at Extended Burnup - FUMEX 1993-1996. Technical Report IAEATECDOC-998, 1998.

[21] L. C. Bernard, J. L. Jacoud, and P. Vesco. An efficient model for the analysis of fission gas release. Journal of Nuclear Materials, 302:125-134, 2002.

[22] G. Pastore, L. P. Swiler, J. D. Hales, S.R. Novascone, D. M. Perez, B. W. Spencer, L. Luzzi, P. Van Uffelen, and R. L. Williamson. Uncertainty and sensitivity analysis of fission gas behavior in engineering-scale fuel modeling. Journal of Nuclear Materials, 456:398-408, 2015.

[23] S. R. Novascone, B. W. Spencer, R. L. Williamson, D. Andrs, J. D. Hales, and D. M. Perez. The effects of thermomechanics coupling strategies in nuclear fuels performance 
simulations. In International Association for Structural Mechanics in Reactor Technology, editor, Transactions of SMiRT-22, San Francisco, CA, August 18-23, 2013.

[24] S.R. Novascone, B.W. Spencer, J.D. Hales, and R.L. Williamson. Evaluation of coupling approaches for thermomechanical simulations. Nuclear Engineering and Design, pages -, 2015. 